ARTICLE

Received 4 Jul 2012 | Accepted 6 Feb 2013 | Published 12 Mar 2013 | Updated 17 Sep 2014

DOI: $10.1038 /$ ncomms 2581

\title{
Perilipin1 promotes unilocular lipid droplet formation through the activation of Fsp27 in adipocytes
}

Zhiqi Sun ${ }^{1, *}$, Jingyi Gong ${ }^{1, \star}$, Han $W u^{1,2}$, Wenyi $X u^{1}$, Lizhen $W^{1}$, Dijin $X u^{1}$, Jinlan Gao ${ }^{1,2}$, Jia-wei Wu ${ }^{1,3}$, Hongyuan Yang ${ }^{4}$, Maojun Yang ${ }^{1,2} \&$ Peng $\mathrm{Li}^{1}$

Mature white adipocytes contain a characteristic unilocular lipid droplet. However, the molecular mechanisms underlying unilocular lipid droplet formation are poorly understood. We previously showed that Fsp27, an adipocyte-specific lipid droplet-associated protein, promotes lipid droplet growth by initiating lipid exchange and transfer. Here, we identify Perilipin1 (Plin1), another adipocyte-specific lipid droplet-associated protein, as an Fsp27 activator. Plin1 interacts with the CIDE-N domain of Fsp27 and markedly increases Fsp27-mediated lipid exchange, lipid transfer and lipid droplet growth. Functional cooperation between Plin1 and Fsp27 is required for efficient lipid droplet growth in adipocytes, as depletion of either protein impairs lipid droplet growth. The CIDE-N domain of Fsp27 forms homodimers and disruption of CIDE-N homodimerization abolishes Fsp27-mediated lipid exchange and transfer. Interestingly, Plin1 can restore the activity of CIDE-N homodimerization-defective mutants of Fsp27. We thus uncover a novel mechanism underlying lipid droplet growth and unilocular lipid droplet formation that involves the cooperative action of Fsp27 and Plin1 in adipocytes.

\footnotetext{
${ }^{1}$ Tsinghua-Peking Center for Life Sciences, Tsinghua University, Beijing 100084, China. ${ }^{2}$ Center for Structural Biology, School of Life Sciences, Tsinghua University, Beijing, 100084 China. ${ }^{3}$ MOE Key Laboratory of Protein Sciences, School of Life Sciences, Tsinghua University, Beijing 100084, China. ${ }^{4}$ School of Biotechnology and Biomolecular Sciences, the University of New South Wales, Sydney, New South Wales 2052, Australia. ${ }^{\star}$ These authors contributed equally to this work.. Correspondence and requests for materials should be addressed to P.L. (email: li-peng@mail.tsinghua.edu.cn).
} 
ipid droplets (LDs) are dynamic cellular organelles that are present in most eukaryotic cells. The LD cores are composed of triglycerides (TAG) and cholesterol esters (CE) and are enclosed by a monolayer of phospholipids ${ }^{1,2}$. LDs serve as energy stores and repositories of fatty acids and sterols, which are used for membrane and hormone synthesis ${ }^{3}$. Recently, LDs have also been found to function in virus packaging ${ }^{4-6}$, intracellular protein storage and protein trafficking ${ }^{7}$. LDs are thought to be derived from the endoplasmic reticulum (ER ${ }^{3}$, and they grow larger by incorporating TAG that is synthesized locally on LD surface ${ }^{8,9}$ or by obtaining TAG from the $\mathrm{ER}^{10-13}$. LD growth may also involve the fusion of small LDs ${ }^{14-17}$. Phosphatidylcholine has a major role in stabilizing the LD surface and preventing LD coalescence, whereas phosphatidic acids may facilitate LD coalescence ${ }^{15,18,19}$. White adipocytes, specialized in energy storage, are characterized by their large unilocular $\mathrm{LDs}^{20}$. LD size correlates with the susceptibility to insulin resistance and diabetes in obese patients ${ }^{21-23}$. However, the molecular mechanisms underlying LD growth and unilocular LD formation in adipocytes are poorly understood.

LDs in different cell types contain unique surface proteins ${ }^{24,25}$. The PAT family proteins, including perilipin (Plin1), adipose differentiation-related protein (ADRP/Plin2) and tail-interacting protein 47 (TIP47/Plin3), are the best-studied LD-associated proteins ${ }^{26-29}$. Plin1 is highly expressed in adipocytes and regulates lipolysis. It associates with the $\mathrm{LD}$ surface through its central domain ${ }^{30-33}$. Both $\mathrm{N}$ - and C-terminal domains of Plin1 are required to block basal lipolysis and mediate hormonestimulated lipolysis. Genetic ablation of Plin1 leads to reduced adiposity, presumably due to elevated basal lipolysis ${ }^{31,32,34,35}$.

The CIDE family proteins Cidea, Cideb and Fsp27/Cidec localize to LDs and are closely linked to the development of metabolic disorders, including obesity, diabetes and liver steatosis $^{36,37}$. Compared with wild-type white adipocytes, which contain unilocular LDs, Fsp27-deficienct white adipocytes accumulate smaller LDs and have reduced lipid storage ${ }^{38,39}$. In contrast, the ectopic expression of Cidea or Fsp27 can significantly increase the LD size ${ }^{40-44}$. Importantly, white adipocytes from a patient containing a homozygous nonsense mutation in Cidec (the human homologue of Fsp27) contain small and multilocular LDs. This patient suffers from partial lipodystrophy, insulin resistance and other metabolic abnormalities ${ }^{45}$. These observations indicate that Fsp27 and Cidea have major roles in controlling LD size in adipocytes. We have recently demonstrated that Fsp27 and Cidea are highly enriched at LD-LD contact sites (LDCSs), where they promote LD fusion and growth by generating a potential lipid diffusion pore at the LDCSs and mediating net lipid transfer from smaller to larger $\mathrm{LDs}^{46}$. Live cell imaging revealed that $\mathrm{LDs}$ indeed grow by a directional lipid transfer process in adipocytes under nearphysiological conditions ${ }^{47,48}$. Interestingly, the rate of Fsp27mediated neutral lipid exchange in adipocytes is $\sim 50$-fold higher than that in preadipocytes overexpressing Fsp27 (ref. 46), indicating the existence of adipocyte-specific activators of Fsp27.

Here, we provide evidence that Plin1 is an adipocyte-specific activator of Fsp27. Plin1 interacts with the CIDE-N domain of Fsp27 and enhances Fsp27-dependent lipid transfer and LD growth. Moreover, Fsp27 CIDE-N domain forms homodimers and this dimerization is crucial for its activity. Interestingly, Plin1 restores the activity of CIDE-N dimerization-defective mutants of Fsp27. These data reveal a novel mechanism for the formation of unilocular LDs in adipocytes through the cooperative action of Fsp27 and Plin1.

\section{Results}

Identification of Plin1 as an Fsp27 activator. To identify potential activators of Fsp27, we co-expressed several LD-associated proteins (Plin1, ADRP/Plin2 and TIP47/Plin3) with Fsp27 in 3T3-L1 pre-adipocytes and examined LD sizes. As demonstrated previously, Fsp27 showed a clear enrichment at the LDCS $^{46}$. When Fsp27-GFP was co-expressed with Plin1, the LDs were significantly larger than those of cells expressing Fsp27 alone (Fig. 1a-c). Notably, Plin1 showed a continuous ring structure on LD surface and was present at the LDCSs, albeit without particular enrichment at the LDCS (Fig. 1a). In contrast, ADRP had no effect on the Fsp27-mediated LD enlargement (Fig. 1a,b). Moreover, Plin 1 did not enhance Cidea activity in promoting LD growth (Fig. 1a-c). When Fsp27 was co-expressed with increasing amounts of Plin1, the population of LDs with diameters larger than $6 \mu \mathrm{m}$ increased, while the number of LDs with diameters smaller than $2 \mu \mathrm{m}$ decreased (Fig. 1d,e), indicating that Plin1 enhances Fsp27's activity in promoting large LD formation in a potential dose-dependent manner.

Among all PAT family proteins, Plin1 expression is highly restricted to adipose tissues. Because lipid exchange is a hallmark of Fsp27-mediated LD growth, we measured the neutral lipid exchange rate in 3T3-L1 adipocytes in which Plin1 was knocked down (Plin1-KD). We depleted $\sim 70 \%$ of Plin1 without significantly affecting the fatty acid binding protein 4 (FABP4) or Fsp27 expression (Fig. 1f). Consistent with previous reports, LD size was significantly reduced in Plin1-KD adipocytes (Supplementary Fig. S1a) $)^{31,32,34,35,38}$. When we followed the fluorescence recovery after photobleaching (FRAP) on adjoining LD pairs in wild-type adipocytes, the fluorescent intensity recovered within $1 \mathrm{~min}$ after each round of photobleaching

\footnotetext{
Figure 1 | Identification of Plin1 as an adipocyte-specific activator of Fsp27. (a) Upper panel: representative images showing that Plin1, but not ADRP/ Plin2, can enhance Fsp27-mediated large LD formation; lower panel, neither Plin1 nor ADRP/Plin2 enhanced Cidea-mediated large LD formation. Empty vector, HA-tagged Plin1, or HA-tagged ADRP/Plin2 were co-expressed with Fsp27-GFP or Cidea-GFP in 3T3-L1 preadipocytes. HA-tagged proteins were stained with HA antibody (blue). LDs were labelled with BODYPI 558/568 C12 fatty acid (BODYPI-FA, red). DAPI (grey)-stained nuclei. Enlarged pictures for splitted channels were shown below and arrowheads point to the LDCSs. Scale bars, $5 \mu \mathrm{m}$. (b) Plin1 enhances Fsp27 but not Cidea's activity in promoting large LD formation in 3T3-L1 preadipocytes. The diameters of the largest LDs in a were measured (mean \pm s.d.; $n \geq 100$ for each group; one-way ANOVA Tukey test, ${ }^{\star \star \star} P<0.001$; NS, no significant difference). (c) Western blot showing similar Fsp27 or Cidea expression levels when co-expressed with Plin1 or ADRP in panel a. GAPDH was used as a loading control. (d) LD size distribution in 3T3-L1 preadipocytes co-expressing Fsp27 and increasing amount of HA-Plin1. Data were collected from $30 \mathrm{GFP}$-positive cells in each group. The percentage of LDs with a diameter $>6 \mu \mathrm{m}$ increased, whereas the percentage of LDs with a diameter $<2 \mu \mathrm{m}$ decreased with increasing expression of Plin1. (e) Western blot showing protein expression levels in d. $\beta$-Actin was used as a loading control. (f) Western blot showing levels of Plin1 in 10-day differentiated 3T3-L1 adipocytes infected with lentivirus containing scramble siRNA (siControl) or siRNA specific for Plin1 (siPlin1). $\beta$-tubulin and FABP4 were used as a loading control and adipocyte differentiation efficiency control, respectively. $(\mathbf{g}, \mathbf{h})$ Representative repeated FRAP experiments showing lipid exchange between contacted LDs in 10-day differentiated siControl (g) or Plin1 knockdown (h) 3T3-L1 adipocytes. MOI in bleached (white circle) and unbleached (blue circle) areas were plotted as the percentage of the initial intensity (right panel). The starting points of photobleaching are indicated by black arrowheads. Scale bars, $5 \mu$ m. (i) Significantly lower lipid exchange rate $\left(n=40\right.$, Mean \pm s.e.m., student t-test, $\left.{ }^{\star \star \star} P<0.001\right)$ in Plin1 knockdown 3T3-L1 adipocytes compared with that of control adipocytes.
} 
(Fig. 1g). However, in Plin1-KD adipocytes, fluorescent signal in the bleached LD recovered slower and failed to reach equilibrium within $1 \mathrm{~min}$ after photobleaching (Fig. 1h), indicating a significantly slower lipid exchange in Plin1-KD adipocytes. Indeed, the lipid exchange rate between contacted LDs in
Plin1-KD adipocytes was $1.28 \pm 0.16 \mu \mathrm{m}^{3} \mathrm{~s}^{-1}$ compared with that of $5.56 \pm 0.62 \mu \mathrm{m}^{3} \mathrm{~s}^{-1}$ in wild-type adipocytes (Fig. 1g-i), representing a $75 \%$ reduction. Therefore, Plin1 functions as an activator of Fsp27, and Plin1 deficiency in adipocytes results in a marked reduction of lipid exchange activity.
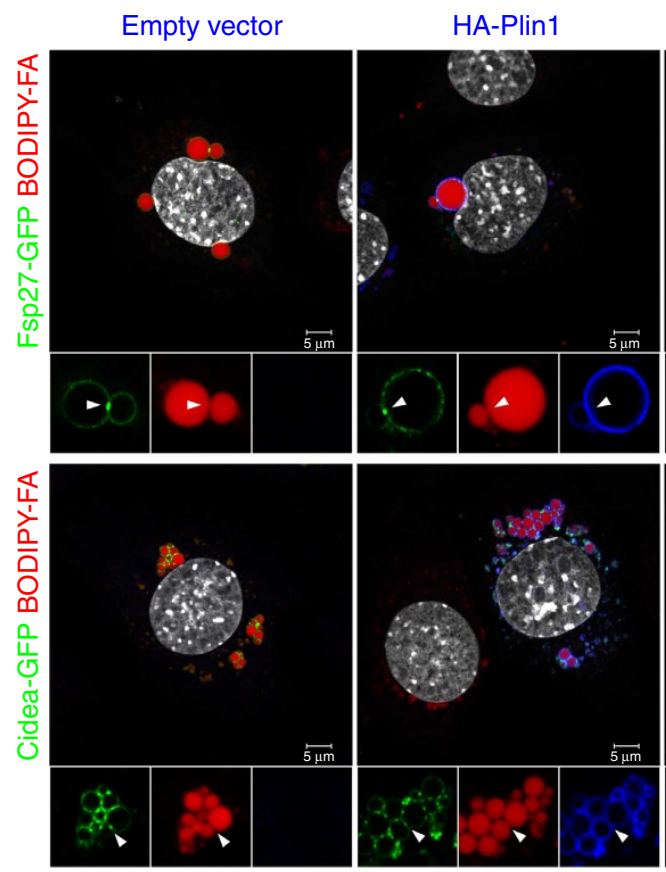

HA-ADRP
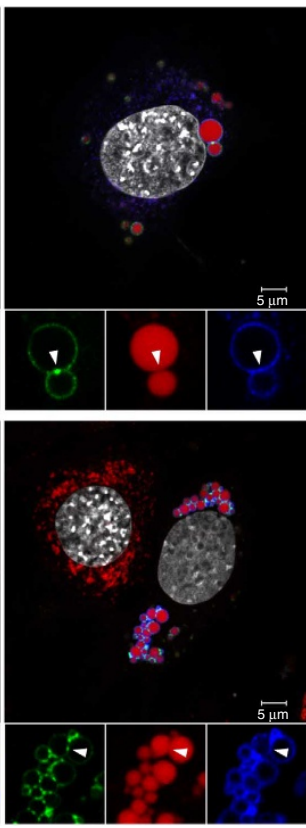

b

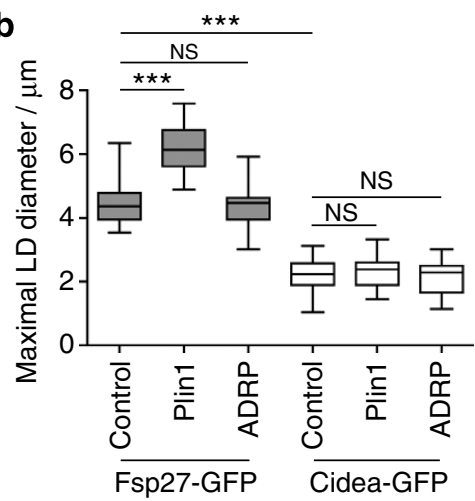

C

Fsp27-GFP Cidea-GFP
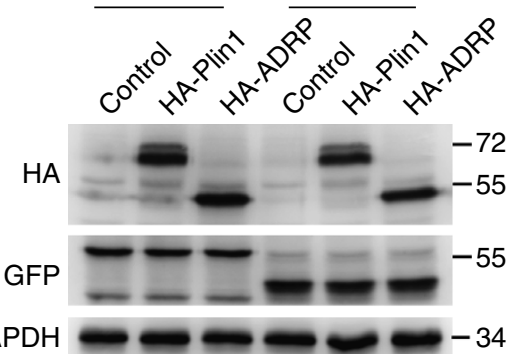

d

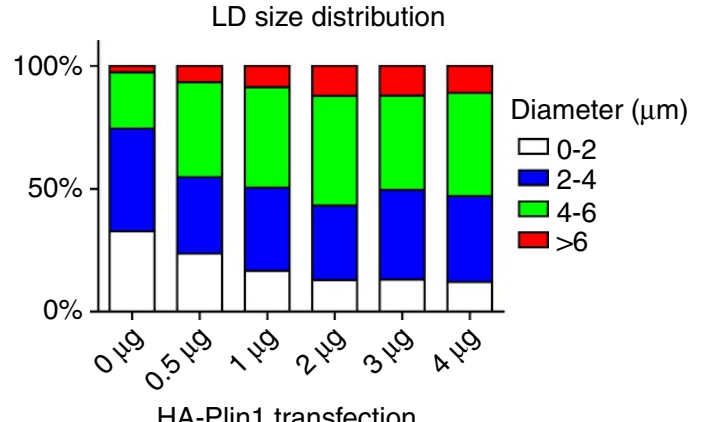

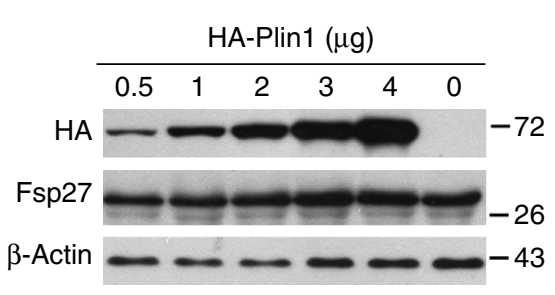

f

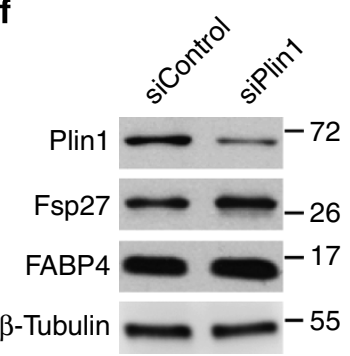

g

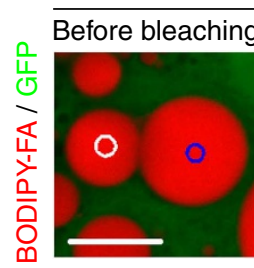

h

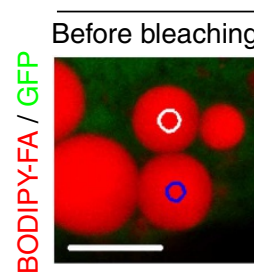

siControl 3T3-L1 adipocyte
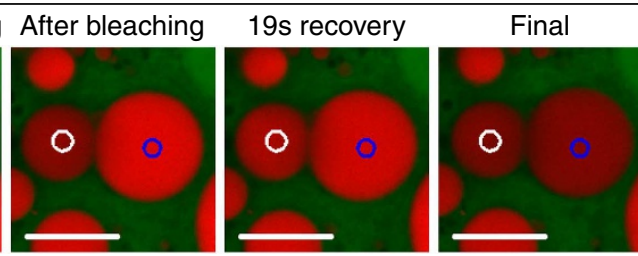

siPlin1 3T3-L1 adipocyte
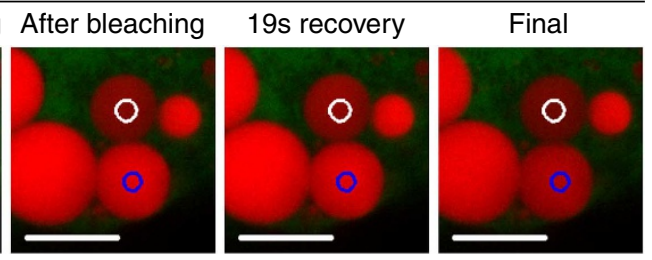
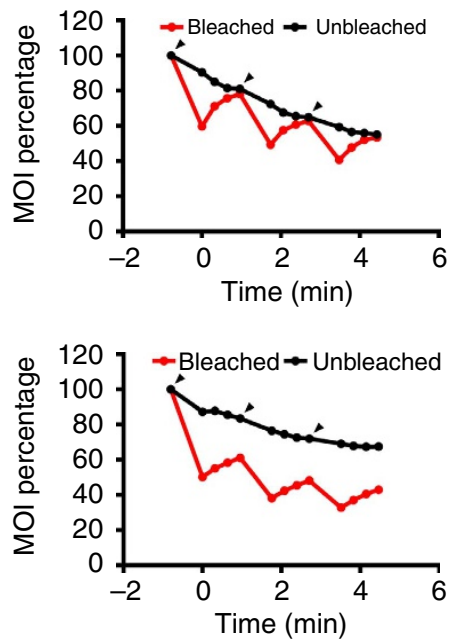

i

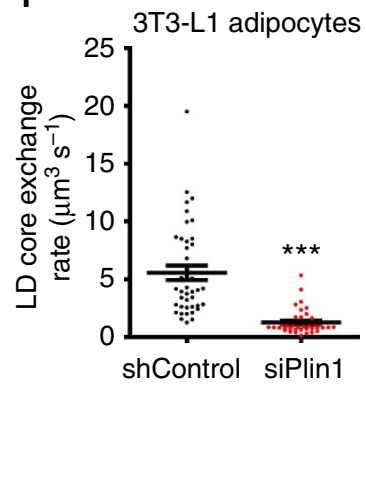


Plin1 interacts with Fsp27. Because both Fsp27 and Plin1 are adipocyte-specific, we investigated whether they form a complex in adipocytes. When endogenous Fsp27 from differentiated 3T3L1 adipocytes was immunoprecipitated, Plin1 but not ADRP was present in the precipitated protein complex; reciprocally, the Fsp27 protein was co-immunoprecipitated with Plin1 but not with ADRP (Fig. 2a,b). Thus, Fsp27 and Plin1 form a complex in adipocytes.

To map the region on Plin1 that mediates its interaction with Fsp27, various Plin1 truncations (Fig. 2c,e) were generated as previously described ${ }^{49}$. The full-length and the central domain (amino acids (a.a.) 233-405), but not the N-terminal (a.a. 1-234) or the C-terminal (a.a. 406-517) domains of Plin1 interacted with Fsp27 (Fig. 2c). Further truncation in the central region of Plin1 revealed that Plin1 (a.a. 1-321) but not Plin1 (a.a. 1-290) or Plin1 (a.a. 319-517) was co-precipitated by Fsp27 (Fig. 2c). Furthermore, the deletion of an acidic residue-rich region between a.a. 292 and 319 (Plin1 $\Delta$ AM) abolished Fsp27-Plin1 interaction (Fig. 2d). Thus, the acidic motif (a.a. 290-321) of Plin1 is indispensable for its interaction with Fsp27. Interestingly, this motif is unique to Plin1 and is not present in other PAT family proteins, consistent with the lack of interaction or functional cooperation between Fsp27 and other PAT proteins (Fig.la and Supplementary Fig. S2a). To identify the region on Fsp27 that mediates its interaction with Plin1, we co-expressed HA-tagged full-length Plin1 with various Flag-tagged Fsp27 truncations. We observed that Fsp27 truncations that contained the CIDE-N domain (a.a. 1-122, a.a. 1-135 and a.a. 38-239) but not the C-terminal region (a.a. 121-239 and a.a. 136-239) pulled down Plin1 (Fig. 2f). Although CIDE family members share a high level of homology, the interaction between Fsp27 and Plin1 is specific, as Plin1 cannot interact with Cidea or Cideb (Fig. 2g).

To quantitatively evaluate the role of Plin1 in promoting Fsp27-dependent LD growth, we arbitrarily defined large LDs as those with a diameter of $2.5 \mu \mathrm{m}$ or larger and counted the percentage of cells containing at least one large LD. When Fsp27 was overxpressed in 3T3-L1 pre-adipocytes, $52 \pm 5 \%$ of Fsp27positive cells contained large LDs (Fig. 2h,i). The co-expression of Plin1 or its central domain (a.a. 233-405) with Fsp27 increased the percentage of cells containing large LDs to $74 \pm 3 \%$ (Fig. $2 \mathrm{~h}, \mathrm{i}$ ). Importantly, Plin1 that lacked the Fsp27 interaction interface (Plin1 $\Delta \mathrm{AM}$ ) did not affect Fsp27-dependent LD enlargement (Fig. 2h,i). The overexpression of Plin1 or Plin1 $\Delta \mathrm{AM}$ alone had no significant effects on the average LD size and neither fulllength nor truncated Plin1 affected Fsp27 expression level (Supplementary Fig. S2b-d). These data suggest that Plin1 and Fsp27 interact with each other and this interaction is required for Plin1 to enhance Fsp27's activity in promoting LD growth.

Plin1 enhances Fsp27-mediated lipid exchange and transfer. We further examined whether Plin1-Fsp27 interaction could regulate Fsp27-mediated neutral lipid exchange by co-expressing Fsp27 and Plin1 or Plin1 $\Delta$ AM in 3T3-L1 preadipocytes. To avoid a possible effect of LD size on lipid exchange, we performed FRAP experiments in adjoining LDs of equivalent size. The lipid exchange rate in cells expressing Fsp27 alone is

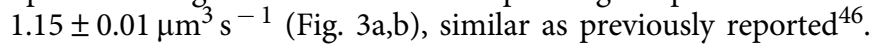
Interestingly, in cells co-expressing Fsp27 and Plin1, there was a drastic fluorescence recovery in the bleached LD in $20 \mathrm{~s}$ (frames in blue box) (Fig. 3a,c). The neutral lipid exchange rate in cells co-expressing Plin1 and Fsp27 was $1.38 \pm 0.12 \mu \mathrm{m}^{3} \mathrm{~s}^{-1}, \sim 10$ fold higher than that in cells expressing Fsp27 alone (Fig. 3e). In contrast, the neutral lipid exchange rate in cells expressing both Plin1 $\triangle \mathrm{AM}$ and Fsp27 was $0.16 \pm 0.01 \mu^{3} \mathrm{~s}^{-1}$, similar to that in cells expressing Fsp27 alone (Fig. 3d,e). Plin1 and Plin1DAM were expressed at similar levels (Fig. 3f), and both were localized to LDs (Supplementary Fig. S2b). Thus, Plin1 can significantly enhance Fsp27-mediated lipid exchange through its interaction with Fsp27.

Next, we examined the lipid transfer in live cells co-expressing Fsp27-GFP and Plin1-Cherry or Plin1 1 AM-Cherry (Fig. 3g). The time required for completing lipid transfer from smaller LDs to larger LDs was much shorter in cells co-expressing Fsp27 and Plin1 than in cells expressing Fsp27 alone (Fig. 3h,i, Supplementary Movies 1 and 2). The net lipid transfer rate was significantly increased in cells co-expressing Fsp27 and Plin1 $\left(0.23 \pm 0.03 \mu \mathrm{m}^{3} \mathrm{~min}^{-1}\right)$ in comparison with cells expressing Fsp27 alone $\left(0.09 \pm 0.03 \mu \mathrm{m}^{3} \mathrm{~min}^{-1}\right.$; Fig. $\left.3 \mathrm{k}\right)$. Plin1 $\triangle \mathrm{AM}$ failed to accelerate lipid transfer when co-expressed with Fsp27 (Fig. 3j,k and Supplementary Movie 3). Overall, these data clearly demonstrate that Plin1 interacts with Fsp27 to enhance Fsp27's activity in promoting lipid exchange and transfer.

Fsp27/Plin1 complex promotes adipocyte giant LD formation. To address the physiological significance of the interaction between Plin1 and Fsp27, we knocked down Plin1 in 3T3-L1 adipocytes (Plin1-KD, Fig. 4a) and observed a dramatically increased basal lipolysis and blunted stimulated lipolysis (Fig. 4b), as previously shown ${ }^{31,32,34,35}$. Consistently, the cellular TAG level in Plin1-KD adipocytes was reduced to $\sim 71 \pm 5 \%$ of the control level (Fig. 4c). To evaluate the contribution of Plin1 to LD size, we measured the diameters of the largest LDs and analysed LD size distribution after three-dimensional (3D) reconstruction. The average diameter of the largest LDs in Plin1-KD adipocytes $(18.6 \pm 0.4 \mu \mathrm{m})$ was dramatically lower than that in control cells $(25.6 \pm 0.8 \mu \mathrm{m}$, Fig. $4 \mathrm{~d}$ and Supplementary Fig. S3a). The size of LDs in wild-type adipocytes varied greatly: the majority of LDs (93.5\%) were smaller than $4 \mu \mathrm{m}$ in diameter; large LDs were easily observable with $\sim 0.7 \%$ of LDs larger than $20 \mu \mathrm{m}$ in diameter (Fig. 4e). Plin1 knockdown resulted in the disappearance of LDs larger than $20 \mu \mathrm{m}$ without affecting the population of $4 \mu \mathrm{m}$ or smaller LDs (94.0\%, Fig. 4e). As expected, Fsp27 knockdown resulted in the accumulation of many small LDs and no LDs with diameters larger than $12 \mu \mathrm{m}$ (Fig. $4 \mathrm{e}$ ).

We next reintroduced siRNA-resistant HA-Plin1 or HAPlin1 $\triangle \mathrm{AM}$ into Plin1-KD adipocytes. The expression levels of Plin1 and Plin1 $\triangle \mathrm{AM}$ were similar to that of control wild-type 3T3-L1 adipocytes, and Plin1 or Plin1 $\triangle \mathrm{AM}$ did not alter the expression of Fsp27 or FABP4 (Fig. 4a). We observed a significantly lower rate of basal lipolysis and a higher rate of stimulated lipolysis (Fig. 4b) and increased TAG content (Fig. 4c) in Plin1-KD cells expressing either wild-type Plin1 or Plin1 $\Delta \mathrm{AM}$ compared with Plin1-KD adipocytes. These data demonstrated that Plin1 and Plin1 $\triangle \mathrm{AM}$ are equally effective in regulating lipolysis. When wild-type Plin1 expression was restored in Plin1$\mathrm{KD}$ adipocytes, the average diameter of the largest LDs was $22.3 \pm 0.7 \mu \mathrm{m}$, much larger than that of Plin1-KD adipocytes (Fig. $4 \mathrm{~d}$ and Supplementary Fig.S3b). In contrast, the average diameter of the largest LDs in Plin1-KD adipocytes re-expressing Plin $1 \Delta \mathrm{AM}$ was $18.4 \pm 0.6 \mu \mathrm{m}$, similar to that in Plin1-KD cells (Fig. 4d and Supplementary Fig.S3b). Notably, the reintroduction of Plin1 but not Plin1 $\triangle \mathrm{AM}$ into Plin1-KD cells significantly restored the population of LDs larger than $20 \mu \mathrm{m}$ (0.5\%; Fig. 4e). Thus, although Plin $1 \Delta \mathrm{AM}$ could restore the inhibition of basal lipolysis in Plin1-KD adipocytes, it failed to rescue Plin1 knockdown in terms of LD enlargement. These data strongly indicate that Plin1 promotes LD growth and unilocular LD formation in adipocytes through its interaction with Fsp27. 
a

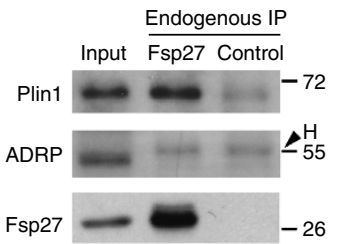

b

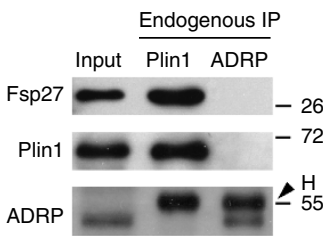

C

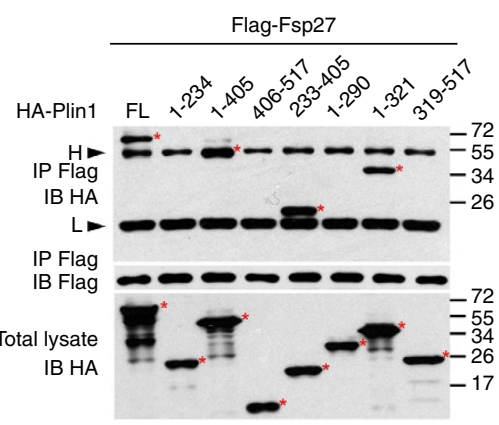

d

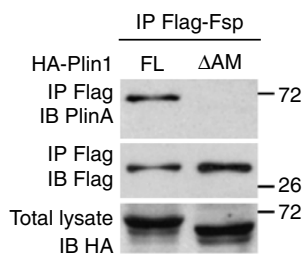

e

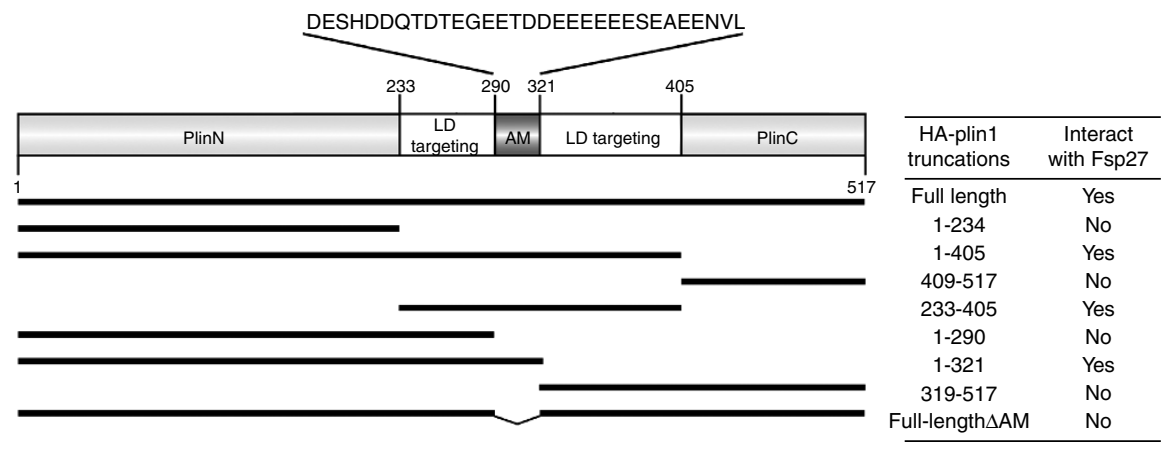

f

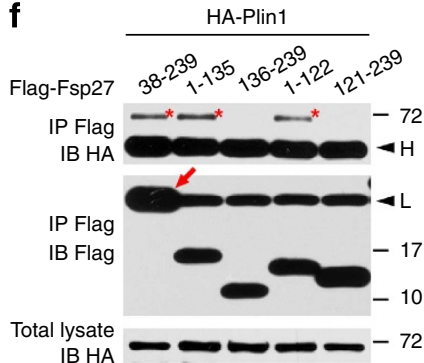

g

HA-Plin 1(233-405)

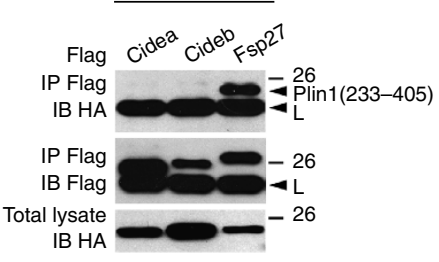

h

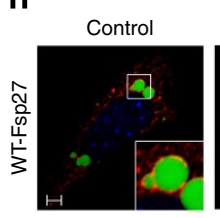

Plin1

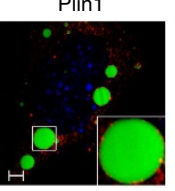

Plin1(233-405)

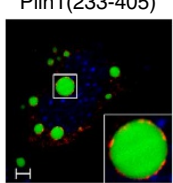

Plin1 AMM

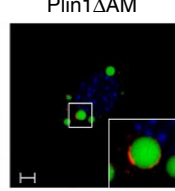

i

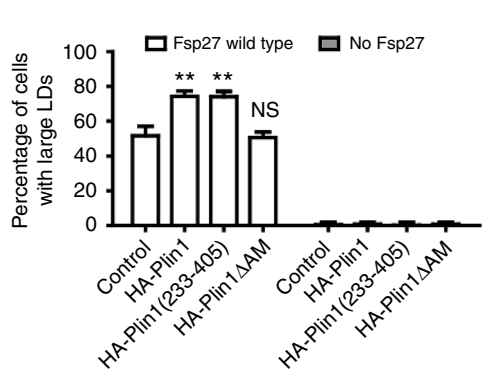

Figure 2 | Plin1 interacts with Fsp27. (a) Fsp27 and Plin1 interact with each other in 3T3-L1 adipocytes. Anti-Fsp27 antibodies were used to immunoprecipitate Fsp27 (IP) from 8-day differentiated 3T3-L1 adipocytes lysates and the presence of Plin1 or ADRP was detected by antibodies against Plin1 or ADRP. Pre-immune serum was used as a negative control. $\mathrm{H}$, heavy chain. (b) Similar to $\mathbf{a}$, except that Plin1 or ADRP/Plin2 were immuneprecipitated (IP) by anti-Plin1 or anti-ADRP antibodies. The presence of Fsp27 in the immunoprecipitated products was detected by anti-Fsp27 antibodies. (c) Acidic motif (a.a. $291-$ 318) of Plin1 mediates its interaction with Fsp27. Full-length Flag-Fsp27 was co-transfected with full-length (FL) or indicated truncations of HA-Plin1 into $293 \mathrm{~T}$ cells. Flag-Fsp27 was immunoprecipitated by anti-FLAG M2-agarose beads. IB, immunoblotting; $H$, heavy chain; L, light chain. Red asterisks mark the positions of Plin1 truncations. Co-immunoprecipitation experiments were performed similarly in $\mathbf{d}, \mathbf{f}$ and $\mathbf{g}$. (d) Deletion of the acidic motif (AM) in the mid-region of Plin1 (Plin1 $\mathrm{AM}$ ) abolishes the Fsp27-Plin1 interaction. (e) Schematic diagram of Plin1 truncations. Right panel summarizes the results from $\mathbf{c}$ and $\mathbf{d}$. (f) The CIDE-N domain (a.a. 38-120) of Fsp27 mediates its interaction with Plin1. Red asterisks mark the HA-Plin1 co-precipitated with indicated Fsp27 truncations. Red arrow points to Fsp27 (a.a. 38-217), which overlaps with the light chain (L). (g) Flag-tagged Fsp27, but not Cidea or Cideb, interacts with Plin1(233-405). (h) Representative images showing that Plin1 can enhance Fsp27's activity in promoting large LD formation through their interaction. Wild-type Fsp27 ( $\mathrm{pCDNA}$ WT-Fsp27) was co-expressed with HA-tagged Plin1 or Plin1(233-405) or Plin1 $\Delta$ AM in 3T3-L1 preadipocytes. Fsp27 proteins are stained with antibody against Fsp27 (red). LDs are stained with BODIPY 493/503 (green). Inserted boxes are enlarged area in the relevant images. Scale bars, $5 \mu \mathrm{m}$. (i) Plin1 enhances Fsp27's activity and the expression of Plin1 alone cannot promote LD growth. Large LDs are defined as LDs with a diameter $\geq 2.5 \mu \mathrm{m}$. The diameter of LDs was measured in total 1,000-1,500 cells from three independent experiments (mean \pm s.d., one-way ANOVA Tukey test, ${ }^{\star \star} P<0.01$; NS, no significant difference). 
CIDE- $N$ forms a homodimer in crystal structural analyses. Because the CIDE-N domain of Fsp27 interacts with Plin1 and has been shown to modulate Fsp 27 activity ${ }^{46}$, we sought to gain further insight into its function by resolving its crystal structure.
The crystal structure of the Fsp27 CIDE-N domain (a.a. 39-119) was solved at $1.93 \AA$ (Supplementary Table S1 and Supplementary Fig. S4). The CIDE-N domain of Fsp27 contains two $\alpha$-helices and four $\beta$-sheets, the whole structure shares an ubiquitin

a

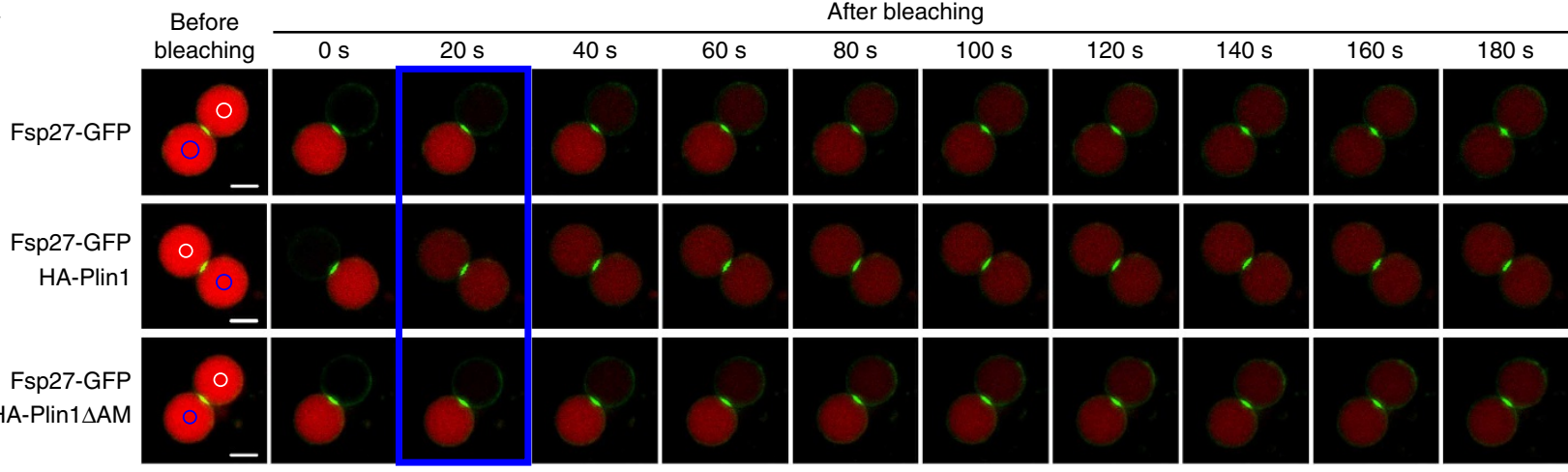

b

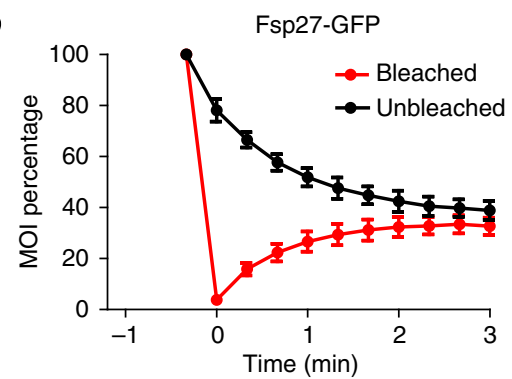

e

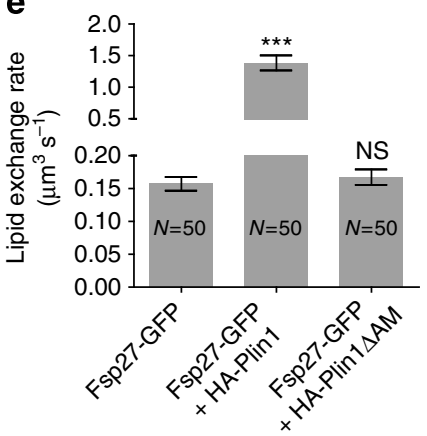

h
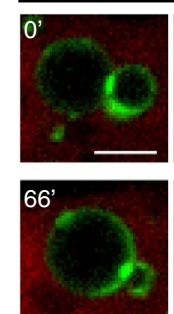

80

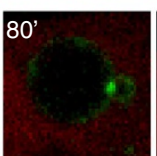

j

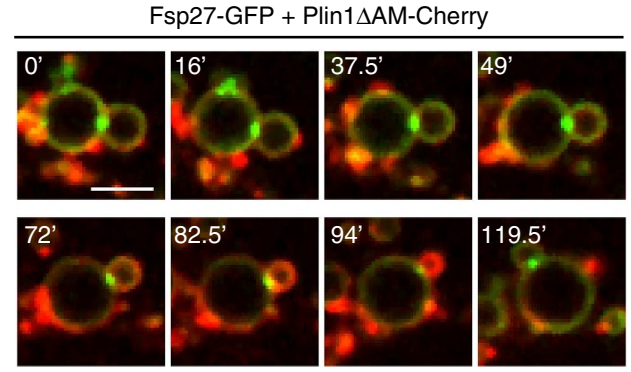

C Fsp27-GFP + HA-Plin1

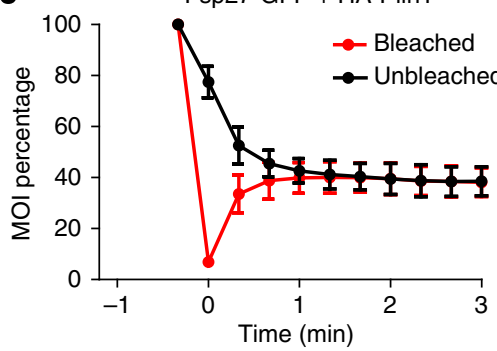

d

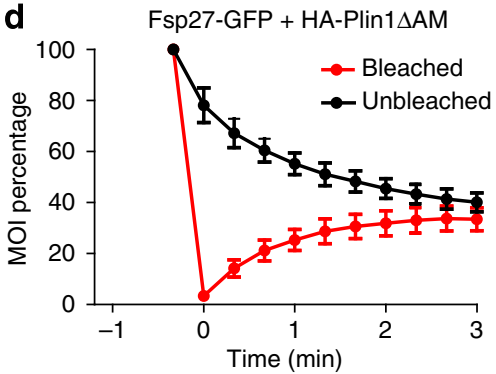

g

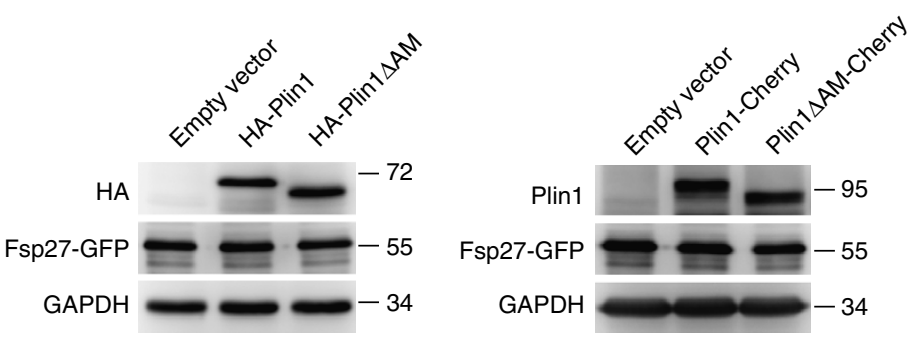


superfamily fold (Fig. 5a). The root mean square deviation between Fsp27 and ubiquitin (PDB: 3M3J) is $2.37 \AA$ (Supplementary Fig. S5a, b). The crystal structure also showed similarities to the solution structures of other CIDE-N domains, with root mean square deviation values of $1.43 \AA$ and $2.54 \AA$ to Cidea (PDB:2EEL) and Cideb (PDB:1D4B), respectively (Supplementary Fig. S5c,d). Structural analyses indicate that Fsp27 CIDE-N domain has the potential to form a dimer in the crystal (Fig. 5a). The putative dimeric structure superimposed well with the N-terminal heterodimeric structure of DFF40/ DFF45 (Supplementary Fig. S5e). The two neighbouring Fsp27 molecules were not aligned symmetrically but were arranged in a yin/yang configuration such that the negatively charged convex portion of the acidic loop on one monomer was wellaccommodated by the positively charged concave surface of the basic region on the adjacent monomer (Fig. 5b and Supplementary Fig. S5f,g). In the structure of Fsp27 CIDE-N domain, the loop between $\beta 3$ and $\alpha 2$, which contains mainly negatively charged residues (E87 and D88) of one molecule interacted with the positively charged residues (R46, R55 and K56) on $\beta 1$ and $\beta 2$ of the adjacent molecule (Fig. 5c). Furthermore, the interface is also characterized by specific electrostatic interactions. For example, R46 forms two ionic bonds with E87 and D88. R55 interacts with the main chain of D88, and K56 contacts E93. Additionally, two $2.8 \AA$ main chain hydrogen bonds are formed between K56 and G57 in one subunit and $\mathrm{I} 91$ in the other (Fig. $5 \mathrm{c}$ ).

To confirm the homodimerization of Fsp27 CIDE-N/CIDE-N, we performed gel filtration analysis. Recombinant Fsp27 CIDE-N (a.a. 39-119) was eluted as a single peak between $27 \mathrm{kDa}$ and $14 \mathrm{kDa}$ in PBS $(137 \mathrm{mM} \mathrm{NaCl})$, close to the predicted molecular weight of the homodimer (Supplementary Fig. S5h). However, in PBS containing $500 \mathrm{mM} \mathrm{NaCl}$, the protein was eluted in a position similar to that of the $14-\mathrm{kDa}$ lysozyme, close to the molecular weight of the monomer $(10 \mathrm{kDa}$; Supplementary Fig. S5i). These data suggest that the CIDE-N domain of Fsp27 forms a dimer, which can be disrupted under high ionic conditions in vitro, consistent with the structural evidence that the CIDE-N dimerizes mainly through electrostatic interaction.

Disruption of CIDE-N dimerization abolishes Fsp27 function. Next, we co-expressed FLAG- and HA-tagged Fsp27 CIDE-N domain and immunoprecipitated FLAG-tagged CIDE-N. HAtagged Fsp27 CIDE-N was co-immunoprecipitated, confirming the homodimerization of Fsp27 CIDE-N (Fig. 6a,b). We then generated various mutations at the CIDE-N/CIDE-N interaction interface based on the crystal structure. The simultaneous substitution of E87Q and D88N (E87Q/D88N) markedly reduced the CIDE-N/CIDE-N interaction (Fig. 6a). Furthermore, the substitution of $\mathrm{E} 86 \mathrm{Q} / \mathrm{E} 87 \mathrm{Q} / \mathrm{D} 88 \mathrm{~N}(\mathrm{QQN})$ or $\mathrm{R} 46 \mathrm{E}$ and $\mathrm{R} 55 \mathrm{E}$ abolished the CIDE-N/CIDE-N interaction (Fig. 6a,b), confirming the interaction revealed by crystal structure. The loss of interaction was not due to protein misfolding, as the QQN mutation did not affect the overall protein folding in CD-spectroscopic analysis (Supplementary Fig. S6a). Interestingly, although the QQN mutation abolished the CIDE-N/CIDE-N interaction, it had little effect on the dimerization of full-length Fsp27, possibly due to the strong interaction between CIDE-C domains (Supplementary Fig. S6b). Bipartite interactions between CIDE proteins are also observed in Cideb $^{50}$. No detectable interaction between the CIDE-N and CIDE-C domains of Fsp27 was observed when the two truncated proteins were co-expressed (Supplementary Fig. S6b).

We then examined the functions of Fsp27 mutants that are defective in CIDE-N/CIDE-N interaction. Despite the similar expression and their enrichment at LDCSs (Supplementary Fig. S6c-e), the Fsp27-GFP mutants defective in the CIDE-N/CIDE-N interaction all showed markedly reduced activity in promoting the large LD formation (Fig. 6c,d). In conclusion, the CIDE-N homodimerization is essential for Fsp27-mediated LD growth.

Plin1 restores CIDE-N dimerization-defective Fsp27 activity. We next tested whether Plin1/Fsp27 heterodimeric and CIDE-N/ CIDE-N homodimeric interactions could co-exist. Plin1 can be effectively pulled down by a homodimerization-defective mutant of the Fsp27 CIDE-N domain (Supplementary Fig. S7a). Furthermore, Plin1 neither enhanced the CIDE-N/CIDE-N interaction nor restored the dimerization of QQN-Fsp27 (Supplementary Fig. S7a). These data suggest that Fsp27 CIDE-N homodimerization and CIDE-N/Plin1 heterodimerization are mediated by independent regions of the CIDE- $\mathrm{N}$ domain. Interestingly, we observed a ring-like arrangement of six lysine residues (K53, K75, K77, K112, K115 and K117) on the surface of the Fsp27 CIDE-N domain that may interact with the acidic motif of Plin1. These residues are not conserved in Cidea or Cideb (Supplementary Fig. S7b,c). Mutations on these lysines weakened the interaction between Fsp27 and Plin1(233-405), suggesting that these residues may contribute to the Fsp27-Plin1 interaction (Supplementary Fig. S7d).

Surprisingly, when the homodimerization-defective QQNFsp27 was co-expressed with full-length Plin1 or Plin1(233-405) but not Plin1 $\triangle \mathrm{AM}$, the percentage of cells that contained large LDs increased significantly (Fig. 7a,b; Supplementary Fig. S2d). Notably, only Plin1 truncations that contained the acidic motif (a.a. 290-321) restored QQN-Fsp27's function (Supplementary Fig. S8a). In addition, Plin1 restored the function of two

Figure 3 | Plin1 enhances Fsp27-mediated lipid exchange and transfer. (a) Representative FRAP images showing that Plin1, but not Plin1 $\Delta$ AM, accelerates Fsp27-mediated neutral lipid exchange. Scale bars, $2 \mu \mathrm{m}$. pEGFP-N1-Fsp27 was co-transfected with pCMV5-HA empty vector (upper panel), pCMV5HA-Plin1 (middle panel) or pCMV5-HA-Plin1 LAM (lower panel) into 3T3-L1 preadipocytes. Cells were incubated with $200 \mu \mathrm{M}$ OA and Bodipy 558/568 C12 fatty acid. Fluorescent lipid signal (red) in one LD was bleached (white circle). Blue box highlighted faster fluorescence recovery in the presence of HAPlin1. (b-d) Fluorescence recovery curves corresponding to FRAP experiments in a. MOI in bleached regions (white circle in a) and unbleached regions (blue circle in a) were plotted as the percentage of the initial intensity. Fluorescent recovery curves are calculated from four independent FRAP experiments (mean \pm s.d.). (e) Lipid exchange rate (mean \pm s.e.m.) in 3T3-L1 preadipocytes expressing indicated proteins. $N$ represents the number of LD pairs used for FRAP data collection. (one-way ANOVA Tukey test; ${ }^{* \star \star} P<0.001$; NS, no significant difference). (f) Western blot showing similar Fsp27-GFP expression and comparable protein levels of HA-Plin1 and HA-Plin1 $\mathrm{AM}$ in a-e. GAPDH was used as a loading control. (g) Western blot showing similar Fsp27-GFP expression and comparable protein levels of Plin1-Cherry and Plin1 $\Delta$ AM-Cherry in h-k. (h) Representative time-lapse images showing lipid transfer from smaller to larger LD in a contacted LD pair with Fsp27 enriched at the LDCS. Fsp27-GFP (green) and Cherry (red) were co-expressed in 3T3-L1 preadipocytes. Scale bars, $4 \mu \mathrm{m}$. (i) Representative time-lapse images showing that Plin1-Cherry accelerates Fsp27-mediated lipid transfer and shortens the duration required for LD fusion when co-expressed with Fsp27-GFP in 3T3-L1 preadipocytes. (j) Representative time-lapse images showing that Plin1 $\Delta$ AMCherry cannot accelerate Fsp27-mediated lipid transfer when co-expressed with Fsp27-GFP in 3T3-L1 preadipocytes. (k) Lipid transfer rate in 3T3-L1 preadipocytes co-expressing Plin1 and Fsp27 is significantly higher than that in cells expressing Fsp27 alone or Plin1 $\Delta$ AM and Fsp27. N represents the number of lipid transfer events used for calculation (mean \pm s.d., one-way ANOVA Tukey test, ${ }^{\star \star \star} P<0.001$ ). 

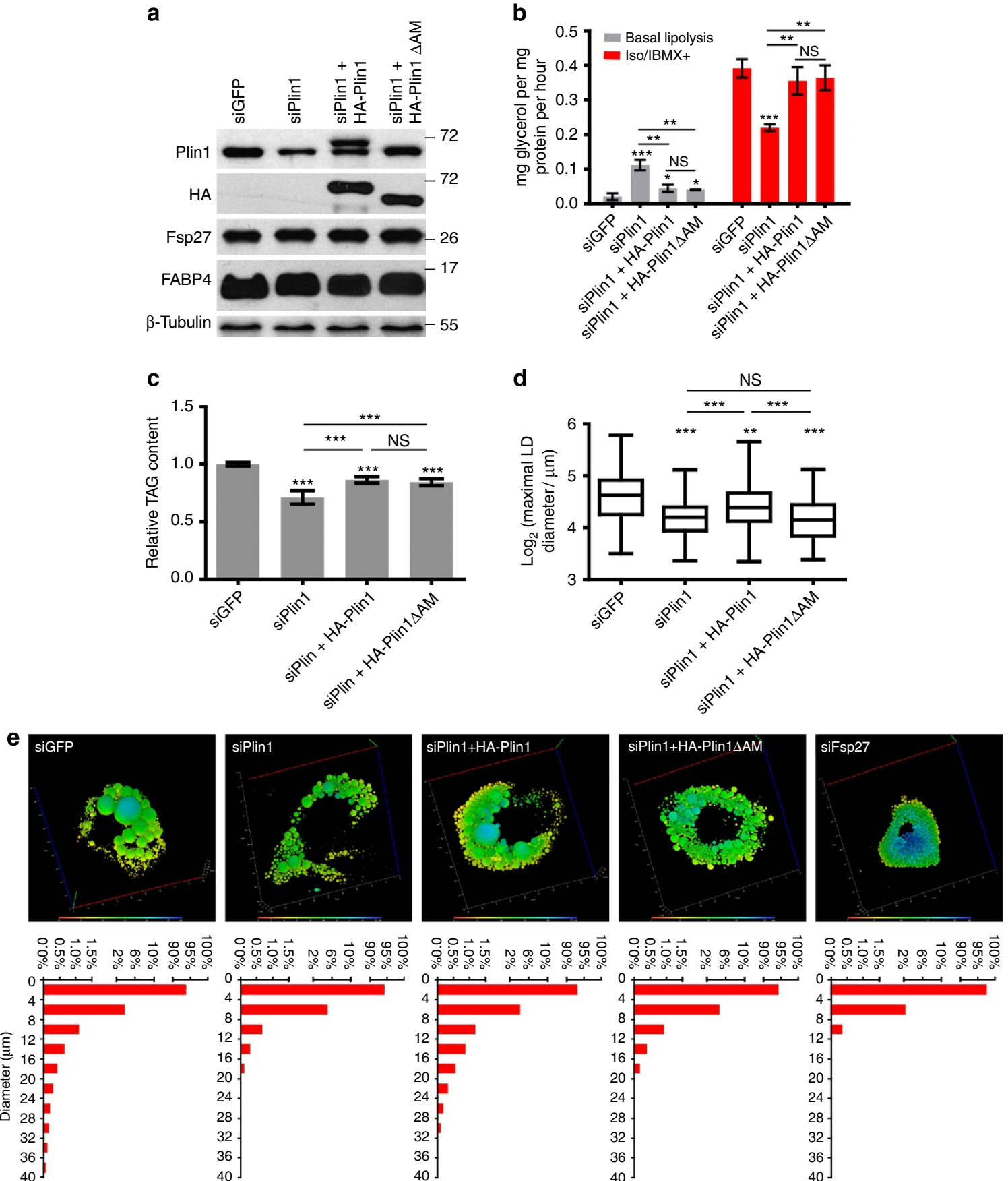

Figure 4 | Fsp27-Plin1 interaction is important for giant LD formation in adipocytes. (a) Western blot showing knockdown of endogenous Plin1 by lentivirus-mediated siPlin1 but not scramble sequence (siControl) and the expression levels of siRNA-resistant HA-tagged Plin1 and Plin1 $\Delta$ AM in 10-day differentiated 3T3-L1 adipocytes. $\beta$-tubulin and FABP were used as a loading control and adipocyte differentiation efficiency control, respectively. (b) Basal and induced lipolysis rates of differentiated adipocytes in $\mathbf{a}$. Mean \pm s.d. were obtained from three independent experiments. Iso, isoproterenol; IBMX, 3-isobutyl-1-methylxanthine (one-way ANOVA Tukey test; ${ }^{\star} P<0.05,{ }^{\star \star} P<0.01,{ }^{\star \star \star} P<0.001$; NS, no significant difference). (c) Relative TAG levels in differentiated adipocytes in $\mathbf{a}$, measured by serum TAG determination kit. Mean \pm s.d. were obtained from three independent experiments (one-way ANOVA Tukey test; ${ }^{\star \star \star} P<0.001$; NS, no significant difference). (d) The diameters of the largest LDs were measured from at least 100 differentiated adipocytes in $\mathbf{a}$ in each group and the log2 (maximal LD diameter) values were shown as box-and-whisker diagram (one-way ANOVA Tukey test; ${ }^{\star \star} P<0.01$, ${ }^{\star \star \star} P<0.001$; NS, no significant difference). (e) Plin1 controls LD size in adipocytes. Representative 3D reconstructed images of differentiated adipocytes in a and Fsp27 knockdown adipocytes (siFsp27) shown in depth-code colour (colour spectrum was shown on the bottom of the image). Lower panel, the LD diameter distribution histogram for each type of adipocytes. Data were collected from 10-15 GFP-positive cells in each group.

other CIDE-N dimerization-defective mutants (R46E, R55E) (Supplementary Fig. S8b). In summary, Plin1 restores the function of QQN-Fsp27 through its interaction with Fsp27.

Consistent with Plin1's compensatory effect on QQN-Fsp27 to induce large LD, the co-expression of Plin1 but not Plin1 $\Delta \mathrm{AM}$ with QQN-Fsp27 restored QQN-Fsp27's activity in mediating lipid exchange, with a rate of $0.12 \pm 0.03 \mu \mathrm{m}^{3} \mathrm{~s}^{-1}$ (Fig. $7 \mathrm{c}-\mathrm{g}$ ). In addition, although QQN-Fsp27 itself cannot promote 

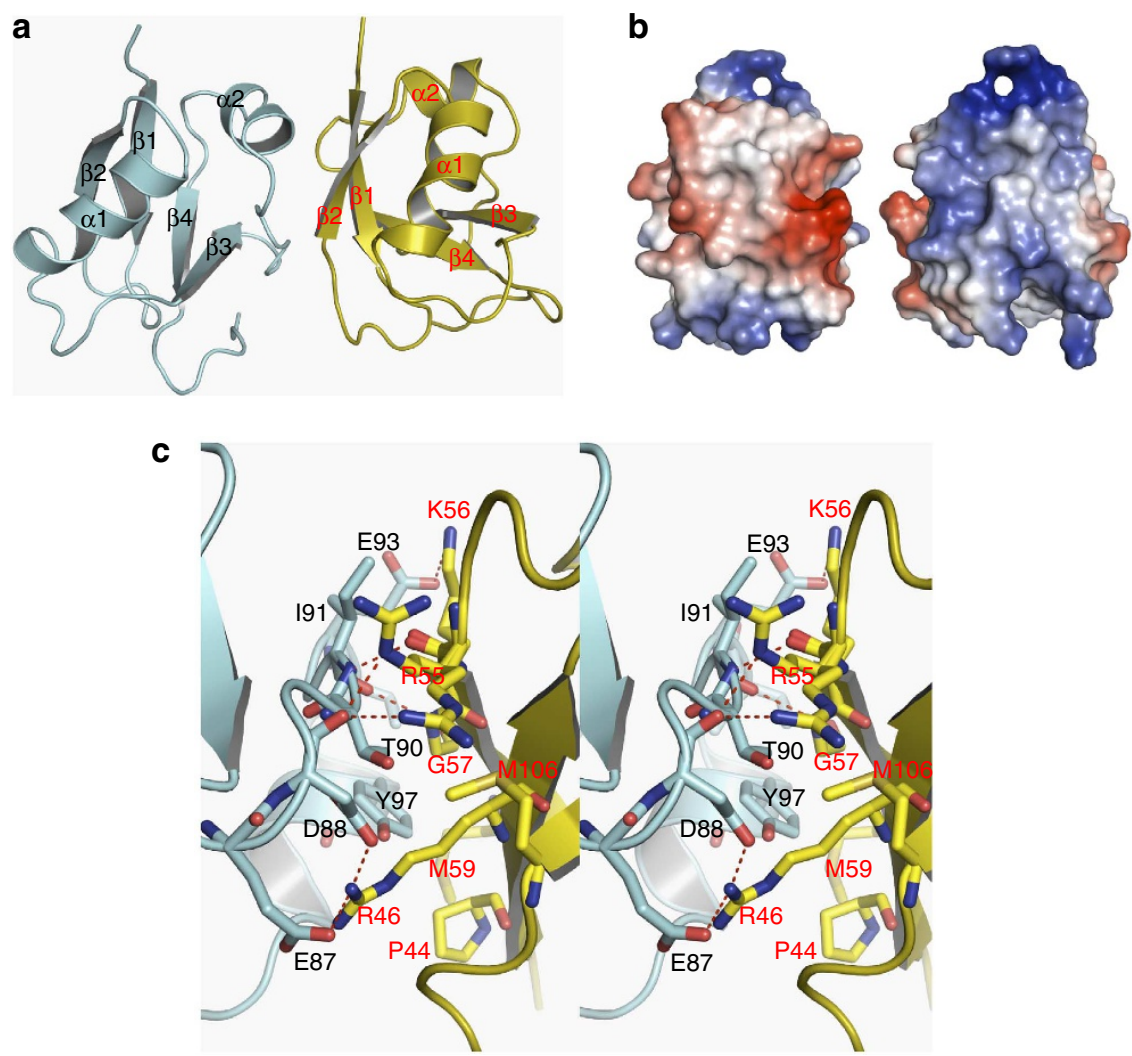

Figure 5 | CIDE-N domain of Fsp27 forms a homodimer. (a) Ribbon diagram showing the crystal structure of CIDE-N homodimer of Fsp27. $\alpha$ and $\beta$ represent the $\alpha$-helices and $\beta$-sheets of the structure. Two interacting molecules are coloured in cyan and yellow, respectively. (b) The electrostatic surface of two interacting molecules. The blue, white and red shadings represent positively charged, neutral and negatively charged surface regions, respectively. (c) Interaction interface of Fsp27 CIDE-N homodimer. Residues in the interaction interface were annotated in red and black colours, respectively. Side chains of contacting residues are shown as sticks. Nitrogen and oxygen atoms are shown in blue and red, respectively. The carbons are coloured in cyan and yellow in the interacted molecules and the hydrogen bonds are shown as dashed lines in red.

lipid transfer, co-expressing QQN-Fsp27 with Plin1 but not Plin1 $\triangle \mathrm{AM}$ resulted in active lipid transfer, with a rate of $0.07 \pm 0.03 \mu^{3} \mathrm{~min}^{-1}$ (Fig. 7h-k and Supplementary Movies 4-6). Overall, despite its inability to restore the interaction of CIDE-N/CIDE-N dimerization-defective mutants, Plin 1 is able to restore their activity in promoting lipid exchange, lipid transfer and LD growth.

\section{Discussion}

Here, we have shown that Plin1, an adipocyte-specific LDassociated protein, interacts with the CIDE-N domain of Fsp27 and enhances Fsp27-dependent lipid transfer and LD growth. In addition, Fsp27 CIDE-N domain forms homodimers and this dimerization is crucial for its activity. Interestingly, Plin 1 can restore the activity of CIDE-N dimerization-defective mutants of Fsp27 in promoting LD growth. These data demonstrate that the CIDE-N domain of Fsp27 controls Fsp27 activity by homodimerizing or recruiting co-factors such as Plin1. Our study thus reveals a novel mechanism for the formation of unilocular LDs in adipocytes.

We have previously shown that the CIDE-C domain of Fsp27 is both necessary and sufficient for the induction of LD growth. However, the presence of the CIDE-N domain significantly increases its activity ${ }^{46}$. This is at least partially due to the CIDE-N homodimerization. Disruption of CIDE-N dimerization diminishes Fsp27's activity in promoting lipid transfer and LD growth without affecting its enrichment at LDCSs. Therefore, the monomeric Fsp27
CIDE-N domain may act as an auto-inhibitory domain to block the function of the CIDE-C domain. As no interaction between CIDE$\mathrm{N}$ and CIDE-C domain was observed, this auto-inhibition is likely due to a conformational effect.

Importantly, Plin1, a key regulator of lipolysis in adipocytes, enhanced Fsp27-mediated lipid transfer and LD growth. This enhancement depends on the interaction between the CIDE-N domain of Fsp27 and a stretch of acidic amino-acid residues in the central domain of Plin1; the deletion of this region from Plin1 (Plin1 $\Delta \mathrm{AM}$ ) disrupted Fsp27-Plin1 interaction and abrogated the enhancement of Fsp27-mediated LD growth. In addition, wildtype Plin1 but not Plin1 $\triangle \mathrm{AM}$ promoted the large LD formation in adipocytes. However, Plin $1 \Delta \mathrm{AM}$ remains capable to regulate basal and stimulated lipolysis. The Fsp27-Plin1 interaction is likely mediated by a ring-like arrangement of six lysine residues, which are not conserved in Cidea or Cideb's CIDE-N domain, explaining the inability of Plin1 to interact with Cidea or Cideb. The functional interaction between Plin1 and Fsp27 is further supported by the observation that Plin 1 restores the activity of CIDE-N homodimerization-defective Fsp27. The crystal structure of the Fsp27 CIDE-N domain indicates that the interfaces mediating homo- and hetero-dimeric interactions are located in different regions. Plin1 may configure the CIDE-N domain of Fsp27 into an active conformation, thereby has a compensatory effect on Fsp27 homodimer-defective mutants. The exact molecular mechanism of how Plin1 enhances Fsp27's activity awaits the crystallographic structural analysis of the Fsp27-Plin1 complex and in vitro biochemical reconstitution. 

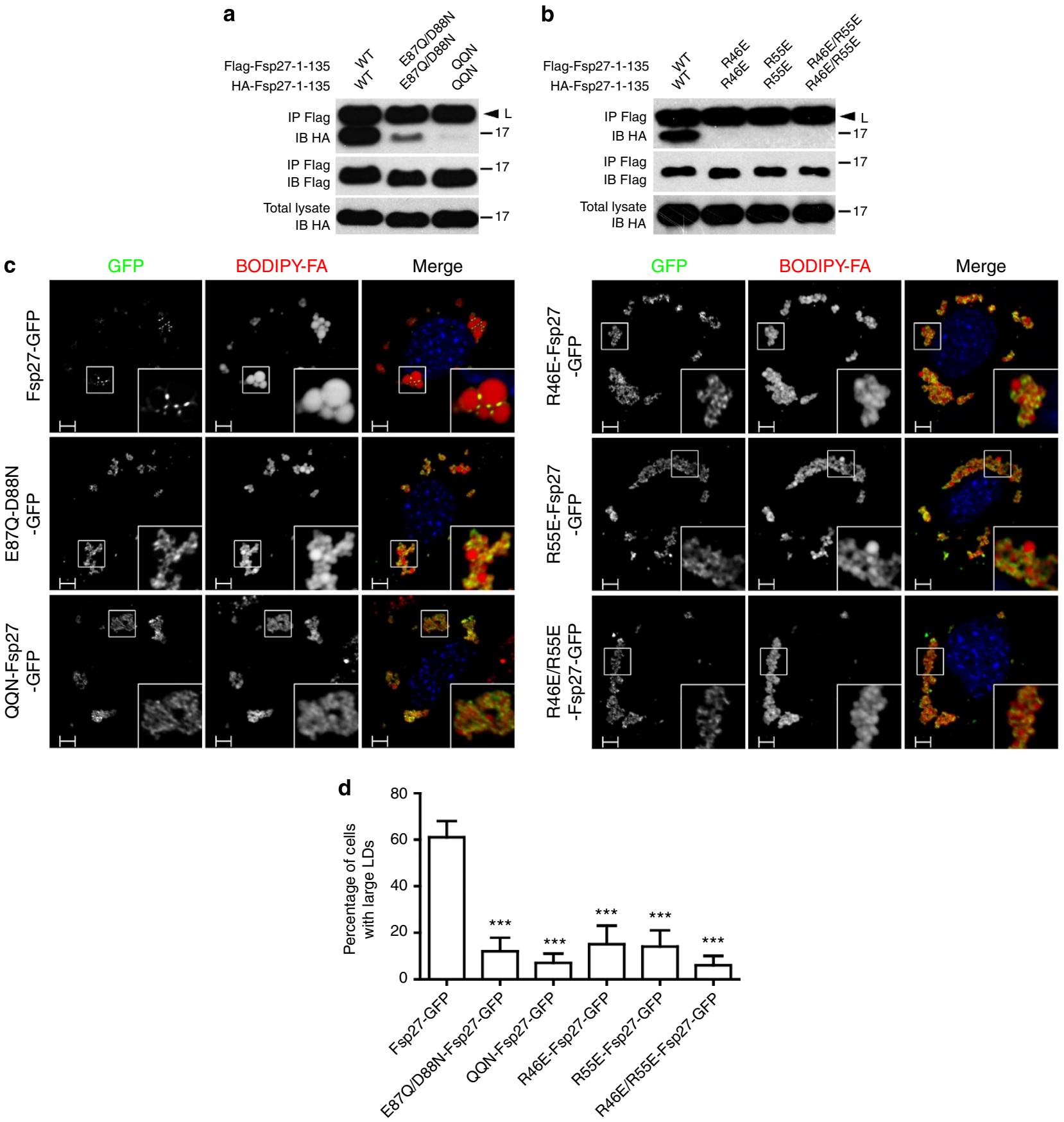

Figure 6 | Disruption of CIDE-N homodimerization abolishes Fsp27-mediated LD growth. (a,b) Mutations at the interaction interface (E87Q/D88N, QQN, R46E, R55E and R46E/R55E) disrupt the CIDE-N homodimerization of Fsp27. Flag and HA-tagged CIDE-N domain of Fsp27 (a.a. 1-135) containing indicated mutations were co-expressed in 293T cells. Anti-Flag M2 beads were used for immunoprecipitation. QQN represents E86Q/E87Q/D88N triple mutations; IP, immunoprecipitation; IB, immunoblot; L, light chain. (c) Representative images showing that CIDE-N homodimerization-defective mutants of full-length Fsp27-GFP (green) fail to induce large LD formation when expressed in 3T3-L1 preadipocytes. LDs were stained with Bodipy 558/568 C12 fatty acid (red). Scale bars, $5 \mu \mathrm{m}$. (d) Quantitative analysis showing that CIDE-N dimerization is critical for Fsp27's activity. Large LDs are defined as LDs with a diameter $\geq 2.5 \mu \mathrm{m}$ in $\mathbf{c} .{ }^{\star \star \star} P<0.001$ represents statistical analysis between wild-type Fsp27 (WT-Fsp27) and mutant forms of Fsp27 (one-way ANOVA Dunnett test, mean \pm s.d., $n \geq 1,000$ ).

We observed a significant increase in lipid exchange and transfer when Plin1 was co-expressed with Fsp27. However, the increases in the rates of lipid transfer and lipid exchange are not proportional (3-fold and 10-fold, respectively). The lipid exchange rate may be mostly determined by the putative pore size, whereas lipid transfer rate is determined by both the LD size and surface tension ${ }^{46}$. Therefore, Plin1 may affect both the pore size and the LD surface tension to modulate Fsp27's activity. Interestingly, brown adipocytes express both Fsp27 and Plin1 but accumulate multilocular small LDs. This might be explained by the high expression of proteins involved in thermogenesis (for example, Ucp1), huge number of mitochondria and active fatty acid oxidation in brown adipocytes. Moreover, oxidative tissuespecific LD proteins (for example, LSDP5 (ref. 51)) may compete 
for LD binding and thus limit the availability of Plin1 for Fsp27. Therefore, the accumulation of differently sized LDs is contingent on specific pathways in different cell types.

We have shown that depleting Plin1 in 3T3-L1 adipocytes increases basal lipolysis and reduces LD size, consistent with previous observations in Plin 1 knockout mice $31,32,34,35$ Moreover, we observed a reduction in basal lipolysis and an increase in LD size when Plin1 was reintroduced into Plin1 knockdown adipocytes. Surprisingly, Plin1 overexpression in mice results in the accumulation of small LDs in white adipocytes ${ }^{52,53}$.

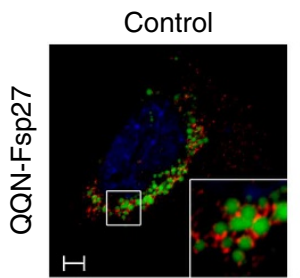

c

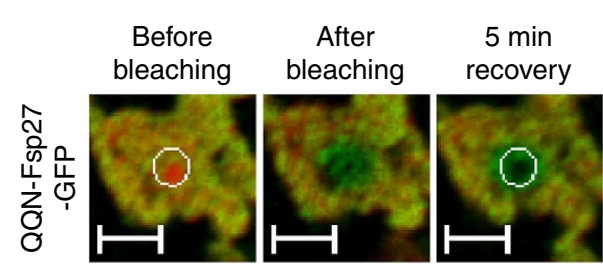

d

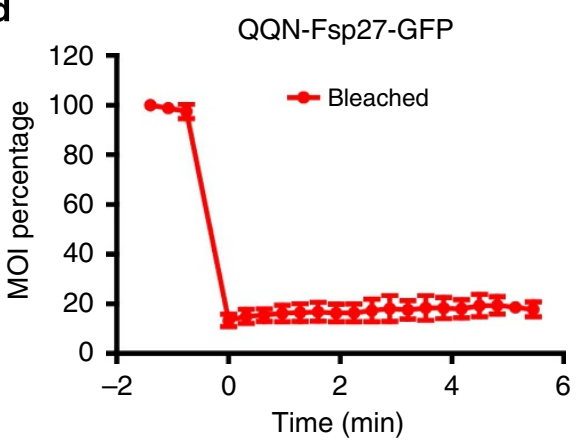

h

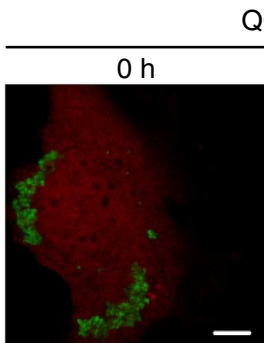

QQN-Fsp27-GFP + Cherry
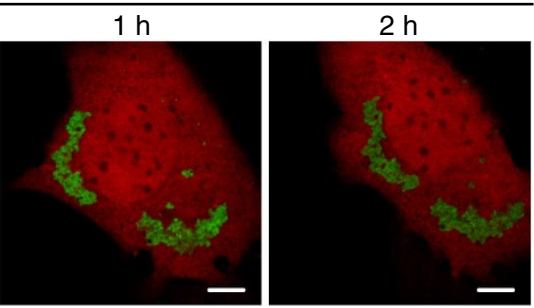

f
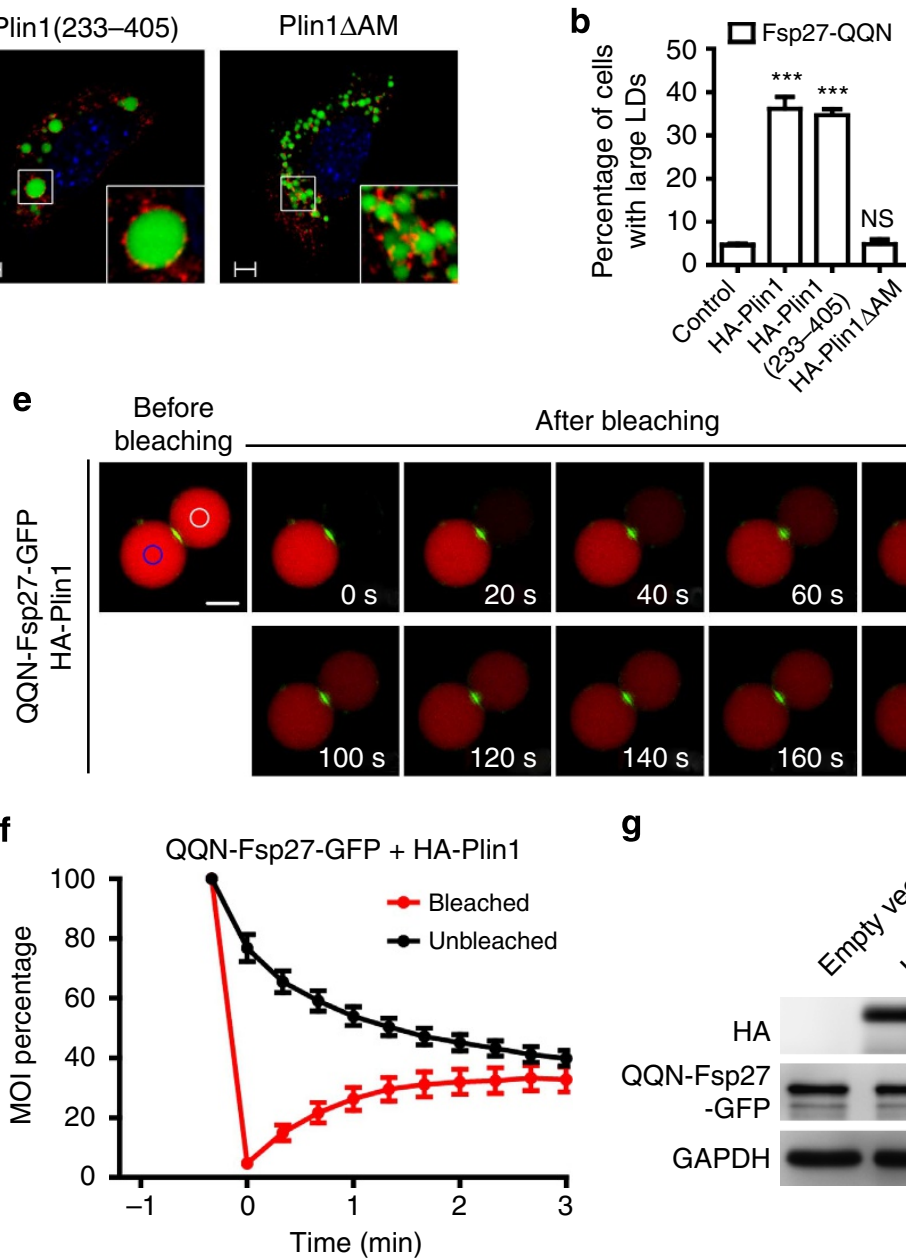

e Before

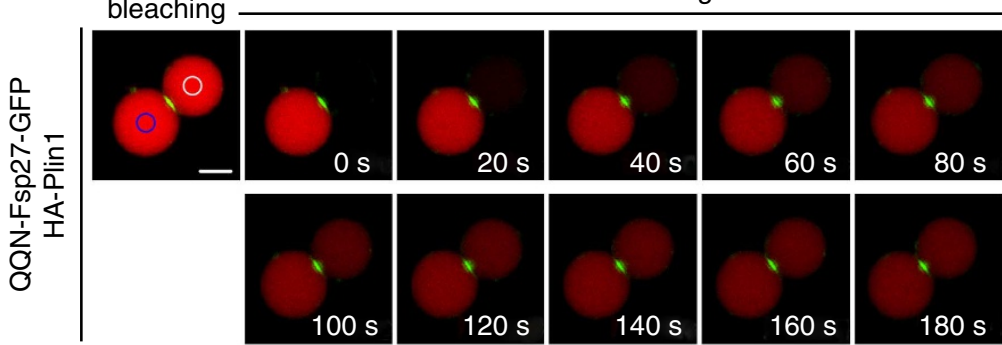

g

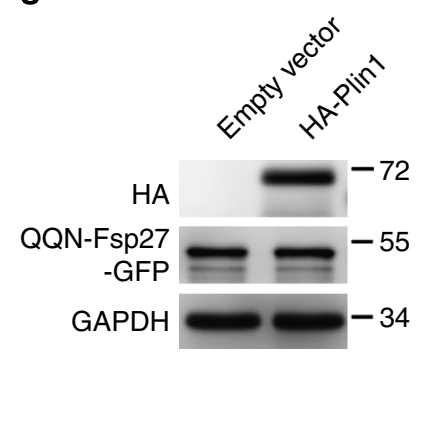

i QQN-Fsp27-GFP + Plin1-Cherry
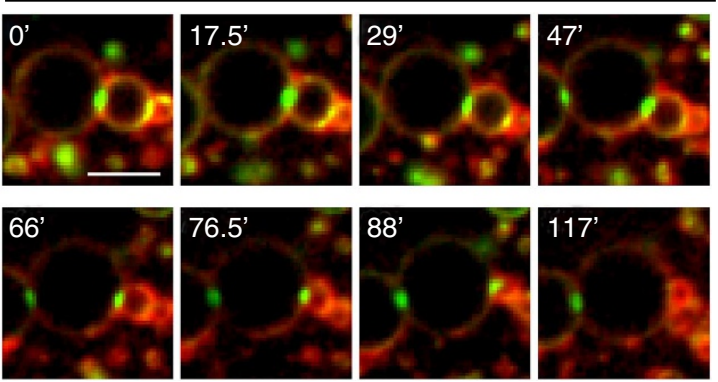

j

QQN-Fsp27-GFP + Plin1 $\triangle$ AM-Cherry
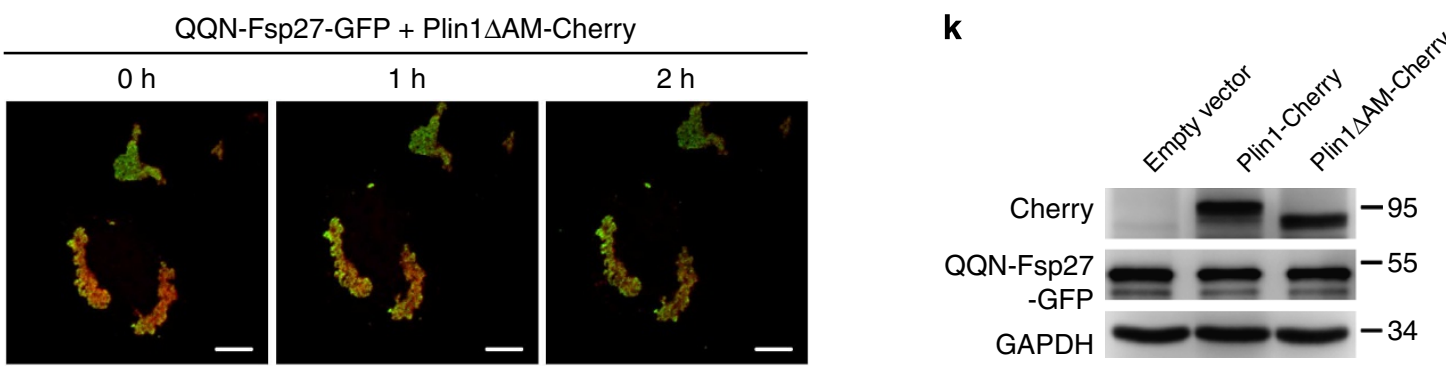

Figure 7 | See next page for figure caption. 
This discrepancy is likely due to the expression levels of Plin1. We noticed that the Plin1 expression level in the white adipose tissue of Plin1 transgenic mice was $\sim 5$-fold higher than that in wildtype mice ${ }^{52,53}$. It is likely that overly high levels of Plin1 in white adipocytes lead to decreased Fsp27 expression and upregulation of genes associated with thermogenesis and fatty acid oxidation, resulting in lean phenotypes ${ }^{52,53}$. When we reintroduced Plin1 into Plin1-KD cells, the levels of Plin1 were similar to that of the endogenous Plin1 in wild-type adipocytes (Fig. 4a). Furthermore, reintroduction of Plin1 did not alter the expression of Fsp27. Therefore, it is important to maintain proper levels of Plin1 in adipocytes to maintain lipid homoeostasis.

Based on the above observations, we propose the following model to explain the role of Fsp27 and Plin1 in promoting unilocular LD formation in adipocytes (Fig. 8). Fsp27 accumulates at the LDCS when two LDs attach each other, creating a pore that specifically allows bidirectional lipid exchange. Several factors, including the size of the lipid transfer pore, the internal pressure difference and LD surface tension could influence the net rate of Fsp27-mediated directional neutral lipid transfer from smaller to larger LDs. The homodimerization of the Fsp27 CIDE$\mathrm{N}$ domain or the formation of the Fsp27-Plin1 heterodimer may promote pore formation/expansion and therefore increase the lipid transfer rate. White adipocytes highly express both Fsp27 and Plin1. The formation of unilocular LDs in white adipocytes could involve multiple lipid transfer events that are initiated by Fsp27 and enhanced by Plin1. Therefore, Fsp27 and Plin1, two adipocyte-specific factors, could act in concert to promote unilocular LD formation in adipocytes.

\section{Methods}

Protein overexpression and purification. The CIDE-N domain of mouse Fsp27 (a.a. 39-119) was subcloned into a modified pRSF-Duet vector containing a His-tag and a TEV protease-recognition site. The recombinant protein was expressed in E. coli strain BL21 and was purified by Ni-NTA-affinity chromatography and gel filtration.

Crystallization and structure determination. Crystals were grown at $18^{\circ} \mathrm{C}$ using the vapor-diffusion method in hanging drop mode by mixing $1.1 \mu \mathrm{l}$ protein with $1.1 \mu \mathrm{l}$ reservoir solution $(0.2 \mathrm{M}$ sodium acetate trihydrate, $0.1 \mathrm{M}$ Tris hydrochloride, $\mathrm{pH} 8.5,28 \%$ polyethylene glycol 4000) and equilibrating against $200 \mu \mathrm{l}$ of reservoir solution. For structure determination, see Supplementary Methods.

Plasmid DNA construction. See Supplementary Methods for details.

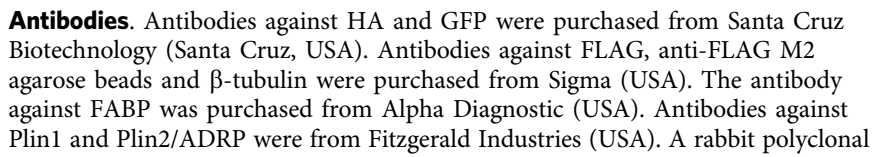

Antibodies. Antibodies against HA and GFP were purchased from Santa Cruz Biotechnology (Santa Cruz, USA). Antibodies against FLAG, anti-FLAG M2 against FABP was purchased from Alpha Diagnostic (USA). Antibodies against Plin1 and Plin2/ADRP were from Fitzgerald Industries (USA). A rabbit polyclonal antibody against mouse Fsp27 was raised against Fsp27 a.a. 1-190 as described previously ${ }^{39}$.

Cell culture and transfection. 293T cells and 3T3-L1 preadipocytes were cultured in Dulbecco's modified Eagle's Medium (DMEM, Invitrogen, USA) containing 10\% fetal bovine serum (Invitrogen, USA). Monolayers of 3T3-L1 preadipocytes were induced to differentiate into mature adipocytes as previously described ${ }^{54}$. Cells were cultured and transfected as previously described ${ }^{46}$.

Immunofluorescent staining. Procedures for immunofluorescent staining, live cell imaging and FRAP analyses were essentially the same as previously described ${ }^{46}$. Images for morphological analysis were acquired either with Zeiss LSM780 laser confocal microscope or under Zeiss $200 \mathrm{M}$ inverted microscope with optical sectioning by Apotome module using a $\times 63$ oil immersion objective ( $\mathrm{VH}$ grid and averaging number 2 were used for optical sectioning).

Live cell imaging. For live cell imaging, showing LD growth and neutral lipidtransfer processes, Fsp27-GFP were overexpressed with indicated proteins in 3T3-L1 preadipocytes. LD pairs for live cell imaging were selected based on the following standards: 1) There is clear and comparable green fluorescent signal enriched at the LDCS. 2) The acceptor LD diameter is $\sim 5 \mu \mathrm{m}(5 \pm 0.5 \mu \mathrm{m})$ and the donor $\mathrm{LD}$ diameter is $\sim 3 \mu \mathrm{m}(3 \pm 0.5 \mu \mathrm{m})$. Images were recorded as a 30 -s time lapse. Lipid transfer rate was calculated as follows: the initial size of the donor LD in diameter was measured at three adjacent starting frames when the ring circle of GFP signal could be clearly visible. The initial size was calculated as the average of the three measurements. The end time point of lipid transfer event was defined when the donor LD shows only punctate structure without measureable size in adjacent five frames. The middle frame of these five frames was documented as the end point of lipid transfer. Lipid transfer rate was calculated by dividing the volume of the donor LD with the duration of the lipid transfer process. A total of 15 independent lipid transfer events in cells expressing Fsp27 in combination with Cherry, Plin1-Cherry or Plin1 $\Delta$ AM-Cherry were acquired, respectively. Lipid transfer rates were calculated accordingly.

FRAP-based lipid exchange assay. 3T3-L1 preadipocytes transfected with indicated plasmids or 8- to 10-day differentiated 3T3-L1 adipocytes were incubated with $200 \mu \mathrm{M}$ OA and Bodipy 558/568 C12 fatty acid (Molecular Probes, no. $\mathrm{D}-3835)$ for $15 \mathrm{~h}$ and changed to fresh medium $1 \mathrm{~h}$ before the experiment. LD pairs with diameter between $4 \mu \mathrm{m}$ and $6 \mu \mathrm{m}$ were selected for bleaching. Selected regions were bleached by 200 interactions at $100 \%$ laser power (543 diode laser), followed by time-lapse scanning with a 20 -s interval. For FRAP analysis, mean optical intensity (MOI) within LD core regions were measured by ImageJ. To obtain fluorescence recovery curves, MOI values were calculated as the percentage of the original fluorescent intensity and plotted using Prism 5.

Three-dimensional reconstruction and measurement. Ten-day differentiated 3T3-L1 adipocytes were incubated with $200 \mu \mathrm{M}$ OA and Bodipy 558/568 C12 fatty acid (red) for $15 \mathrm{~h}$, fixed and subjected to image analysis. Z-stack images were acquired at $0.37 \mu \mathrm{M}$ per section under LSM710 microscope. Images of representative cells were shown in depth-code colour. Volume of each of the LDs was analysed with 3D objects counter in ImageJ. LD volume information from 10 to 15 cells was included for each LD diameter distribution histogram.

Lentivirus preparation and generation of stable cell lines. A DNA fragment encoding a siRNA specific for Plin1 ( $5^{\prime}$-GATCCGGGTGTTACGGATAACGTGTTCAAGAGACACGTTATCCGTAACACCCTTTTTTGGAAA-3', 5'-AGCTTTTCCAAAAAAGGGTGTTACGGATAACGTGTCTCTTGAACAC

Figure 7 | Plin1 restores the activity of CIDE-N dimerization-defective mutant. (a) Representative images showing that Plin1 can restore the activity of QQN-Fsp27 in promoting LD enlargement in 3T3-L1 preadipocytes. Full-length CIDE-N homodimerization-defective Fsp27 (pCDNA-QQN-Fsp27) was co-expressed with HA-tagged Plin1, Plin1(233-405) or Plin1 $\triangle$ AM in 3T3-L1 preadipocytes. Fsp27 was stained with antibody against Fsp27 (red) and LDs are stained with Bodipy 493/503 (green). Scale bars, $5 \mu \mathrm{m}$. (b) Plin1 restores the activity of QQN-Fsp27 in 3T3-L1 preadipocytes in promoting large LD formation. Large LD is defined as the LD with a diameter $\geq 2.5 \mu \mathrm{m}$ in (a). One-way ANOVA Tukey test, ${ }^{\star \star \star} P<0.001$ and NS (no significant difference) represents statistical analysis between QQN-Fsp27 alone and its co-expression with Plin1 or Plin1 truncations. (c,d) No neutral lipid diffusion among LD clusters in 3T3-L1 preadipocytes expressing QQN-Fsp27-GFP. Neutral lipid signal (red) in the white circle was bleached (c), scale bars, $2 \mu \mathrm{m}$. (d) MOI in bleached region was plotted as the percentage of the initial intensity from four independent experiments (mean \pm s.d., lower panel). (e,f) Plin1 restores the lipid exchange activity of QQN-Fsp27 when co-expressed in 3T3-L1 preadipocytes. (e) Neutral lipid signal (red) in one LD was bleached (white circle). Scale bars, $2 \mu \mathrm{m}$. (f) $\mathrm{MOI}$ in bleached region (white circle) and unbleached region (blue circle) were plotted as the percentage of the initial intensity from four independent experiments (mean \pm s.d., lower panel). (g) Western blot showing similar QQN-Fsp27-GFP expression in the absence or presence of HAPlin1. GAPDH was used as a loading control. (h) Representative time-lapse images showing that QQN-Fsp27-GFP cannot initiate lipid transfer. pEGFP-N1QQN-Fsp27 was co-transfected with pmCherry-N1 into 3T3-L1 preadipocytes. Scale bars, $4 \mu \mathrm{m}$. (i,j) Representative time-lapse images showing that Plin1 but not Plin1 $\triangle$ AM can restore the activity of QQN-Fsp27 in promoting lipid transfer when co-expressed in 3T3-L1 preadipocytes. Scale bars, $4 \mu \mathrm{m}$. (k) Western blot showing similar QQN-Fsp27-GFP expression and comparable protein levels of Plin1-Cherry and Plin1 $\Delta$ AM-Cherry in $\mathbf{h}-\mathbf{j}$ 


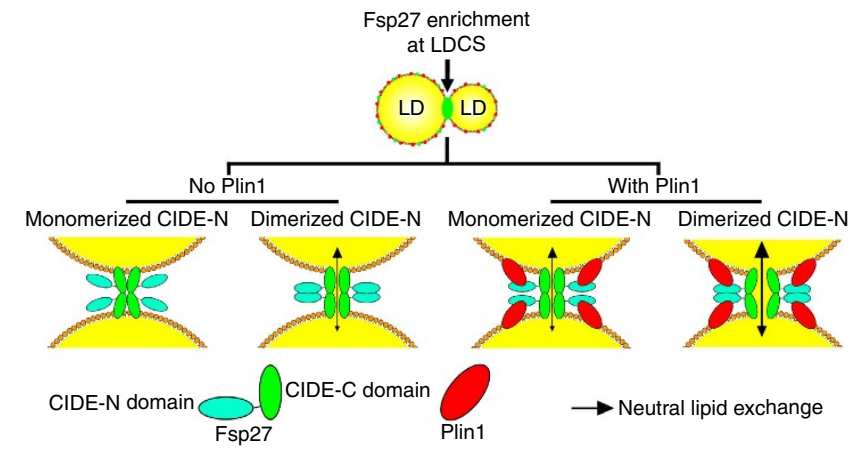

Figure 8 | Model for the role of Plin1 in regulating Fsp27-mediated lipid transfer and LD growth. When Fsp27 is enriched at the LD contact site, it may adapt two conformations: the monomeric conformation that is inactive and the homodimeric conformation that is active in promoting lipid exchange, net lipid transfer and LD growth. Plin1 interacts with Fsp27 at the CIDE-N domain and changes the conformation of Fsp27, resulting in a restoration of the activity of CIDE-N monomeric inactive Fsp27 and an enhancement of the activity of dimeric Fsp27.

GTTATCCGTAACACCCG-3') was inserted into pSilencer2.1-U6 hygro vector (Ambion, USA), subcloned into FG12 expression vector between EcoRI and Hind III sites. Lentivirus packaging and stable knockdown cell line generating were performed as previously described ${ }^{46}$. Infection efficiency was monitored by the signal of GFP, which is co-integrated into genome with siRNA expression module. Infection efficiency reached $95 \%$. Knockdown efficiency was analysed by western blot analysis.

Production and infection of recombinant adenovirus. N-terminal HA-tagged siRNA-resistant (containing three nucleotide mutations in the siRNA-binding site) full-length Plin1 or its acidic motif deletion (Plin1 $\triangle \mathrm{AM}$ ) was subcloned into pShuttle-CMV vector. Adenovirus were generated and amplified in AD293 cells in AdEasy-1 system according to manufacturer's instruction (Stratagene, USA). The titre of recombinant adenovirus was determined by anti-HA immunostaining. Seven-day differentiated 3T3-L1 adipocytes that already contained lentivirus against siPlin1 or siGFP were trypsinized, seeded and infected with recombinant adenovirus at a titre of 100 multiplicity of infection after $12 \mathrm{~h}$. Cells were used for experiments 3 days after infection. Infection efficiency was monitored by anti-HA immunostaining and was higher than $95 \%$.

Lipolysis and cellular TAG measurement. Lipolysis and the cellular TAG were determined as previously described ${ }^{39,54}$. Briefly, for lipolysis assay, 10-day differentiated 3T3-L1 adipocytes were washed three times with pre-warmed PBS and then incubated in serum-free medium containing $1 \%$ fatty acid-free BSA in the presence or absence of $10 \mu \mathrm{M}$ isoproterenol and $10 \mu \mathrm{M}$ IBMX. The amount of glycerol released into culture medium at $0,10,30,60,120$ and 240 min was measured by Free Glycerol determination kit (Sigma). Experiments were repeated in triplicates. The levels of released glycerol showed a linear increase within $120 \mathrm{~min}\left(r^{2}>0.95\right)$. The rate of basal and induced lipolysis was calculated from the linear fitting.

For cellular TAG level determination, lipids were extracted from 10-day differentiated 3T3-L1 adipocytes as previously described ${ }^{55}$, dissolved in isopropanol and determined by serum TAG determination kit (Sigma). TAG levels were normalized to the protein concentration of each sample.

Image processing. All frames in the same time-lapse or FRAP experiments were adjusted in parallel in levels or contrast through Image Pro6.0 or Carl Zeiss Zen 2009. Images were exported out in 16-bit TIFF format. Further processing of single image (for example, amplifying certain region) was performed in Photoshop CS2. Movies were exported from Carl Zeiss Zen 2009 and time stamps were incorporated by ImageJ.

LD size measurement and evaluation. For quantitative analysis of cells containing large LDs (Figs 2h,i, 6d and 7a,b), 3T3-L1 preadipocytes transfected with indicated plasmids were fixed and stained with Bodipy 493/503 (green). DsRed-N2 was co-expressed as a transfection control. The diameter of LDs was measured in a total of 1,000-1,500 cells from three independent experiments for each column. The percentage of cells containing at least one LD larger than $2.5 \mu \mathrm{m}$ in diameter was calculated.

To measure the LD size in differentiated 3T3-L1 adipocytes, cells that were infected with corresponding recombinant adenovirus for 3 days were fixed and stained with anti-HA antibodies. Images for HA and GFP double-positive adipocytes were acquired and the diameter of the largest $\mathrm{LD}$ in each cell was measured by AxioVision (Carl Zeiss). About 100 cells for each condition were counted for statistical analysis. Cells with the largest LD smaller than $10 \mu \mathrm{m}$ in diameter were not included in the calculation.

Co-immunoprecipitation. Co-immunoprecipitation was performed as previously described $^{56}$. For immuoprecipitation of endogenous proteins, 8-day differentiated 3T3-L1 adipocytes were washed twice by PBS, treated with PBS containing $2 \mathrm{mM}$ Dithiobis-succinimidylpropionate (DSP, Pierce) for $30 \mathrm{~min}$, and lysed in IP buffer ( $25 \mathrm{mM}$ HEPES, pH7.4, $150 \mathrm{mM} \mathrm{NaCl}, 1 \%$ Triton X-100) by sonication. Antibodies against Fsp27, Plin1, Plin2/ADRP or pre-immune serum were used for immunoprecipitation.

Statistics. Data from at least three independent experiments were subjected to statistical analysis and plotted using Microsoft Office Excel 2007 or Graphpad Prism 5.0. Results were reported either by mean \pm s.e.m. or mean \pm s.d. as indicated in the figure legend. Student's $t$-test was used for two condition comparison. One-way ANOVA with either Tukey or Dunnett multiple comparison was used for multiple conditions comparison; the $P$-value was multiplicity adjusted.

Calculation of neutral lipid exchange rate. Detailed algorithm of the calculation of lipid exchange rate was described by Gong et al. ${ }^{46}$ and in Supplementary Methods. We selected a LD pair linked by one typical LDCS. Digital gain and laser power were set to avoid overexposure and ensure accurate quantification of fluorescence. The neutral lipid core of one LD was photo-bleached and the MOIs of both bleached and unbleached LD were measured with ImageJ.

\section{References}

1. Farese, Jr R. V. \& Walther, T. C. Lipid droplets finally get a little R-E-S-PE-C-T. Cell 139, 855-860 (2009).

2. Krahmer, N., Guo, Y., Farese, Jr R. V. \& Walther, T. C. SnapShot: Lipid Droplets. Cell 139, 1024-1024 el (2009).

3. Martin, S. \& Parton, R. G. Lipid droplets: a unified view of a dynamic organelle. Nat. Rev. Mol. Cell. Biol. 7, 373-378 (2006).

4. Samsa, M. M. et al. Dengue virus capsid protein usurps lipid droplets for viral particle formation. PLoS Pathog. 5, e1000632 (2009).

5. Miyanari, Y. et al. The lipid droplet is an important organelle for hepatitis $\mathrm{C}$ virus production. Nat. Cell. Biol. 9, 1089-1097 (2007).

6. Cheung, W. et al. Rotaviruses associate with cellular lipid droplet components to replicate in viroplasms, and compounds disrupting or blocking lipid droplets inhibit viroplasm formation and viral replication. J. Virol. 84, 6782-6798 (2010).

7. Cermelli, S., Guo, Y., Gross, S. P. \& Welte, M. A. The lipid-droplet proteome reveals that droplets are a protein-storage depot. Curr. Biol. 16, 1783-1795 (2006).

8. Kuerschner, L., Moessinger, C. \& Thiele, C. Imaging of lipid biosynthesis: how a neutral lipid enters lipid droplets. Traffic 9, 338-352 (2008).

9. Stone, S. J. et al. The endoplasmic reticulum enzyme DGAT2 is found in mitochondria-associated membranes and has a mitochondrial targeting signal that promotes its association with mitochondria. J. Biol. Chem. 284, 5352-5361 (2009).

10. Gross, D. A., Zhan, C. \& Silver, D. L. Direct binding of triglyceride to fat storage-inducing transmembrane proteins 1 and 2 is important for lipid droplet formation. Proc. Natl Acad. Sci. 108, 19581-19586 (2011).

11. Kadereit, B. et al. Evolutionarily conserved gene family important for fat storage. Proc. Natl Acad. Sci. USA 105, 94-99 (2008).

12. Bulankina, A. V. et al. TIP47 functions in the biogenesis of lipid droplets. J. Cell. Biol. 185, 641-655 (2009).

13. Yang, H., Galea, A., Sytnyk, V. \& Crossley, M. Controlling the size of lipid droplets: lipid and protein factors. Curr. Opin. Cell. Biol. 24, 509-516 (2012).

14. Fei, W. et al. Fld1p, a functional homologue of human seipin, regulates the size of lipid droplets in yeast. J. Cell. Biol. 180, 473-482 (2008).

15. Guo, Y. et al. Functional genomic screen reveals genes involved in lipid-droplet formation and utilization. Nature 453, 657-661 (2008).

16. Bostrom, P. et al. SNARE proteins mediate fusion between cytosolic lipid droplets and are implicated in insulin sensitivity. Nat. Cell. Biol. 9, 1286-1293 (2007).

17. Murphy, S., Martin, S. \& Parton, R. G. Quantitative analysis of lipid droplet fusion: inefficient steady state fusion but rapid stimulation by chemical fusogens. PLoS One 5, e15030 (2010).

18. Fei, W. et al. A role for phosphatidic acid in the formation of 'supersized' lipid droplets. PLoS Genet. 7, e1002201 (2011).

19. Krahmer, N. et al. Phosphatidylcholine synthesis for lipid droplet expansion is mediated by localized activation of CTP:phosphocholine cytidylyltransferase. Cell Metab. 14, 504-515 (2011).

20. Rosen, E. D. \& Spiegelman, B. M. Adipocytes as regulators of energy balance and glucose homeostasis. Nature 444, 847-853 (2006). 
21. McLaughlin, T. et al. Enhanced proportion of small adipose cells in insulinresistant vs insulin-sensitive obese individuals implicates impaired adipogenesis. Diabetologia 50, 1707-1715 (2007).

22. Guilherme, A., Virbasius, J. V., Puri, V. \& Czech, M. P. Adipocyte dysfunctions linking obesity to insulin resistance and type 2 diabetes. Nat. Rev. Mol. Cell. Biol. 9, 367-377 (2008).

23. DeFronzo, R. A. Dysfunctional fat cells, lipotoxicity and type 2 diabetes. Int. J. Clin. Pract. Suppl. 9-21 (2004).

24. Walther, T. C. \& Farese, Jr R. V. The life of lipid droplets. Biochim. Biophys Acta 1791, 459-466 2009.

25. Wolins, N. E., Brasaemle, D. L. \& Bickel, P. E. A proposed model of fat packaging by exchangeable lipid droplet proteins. FEBS Lett. 580, 5484-5491 (2006).

26. Bickel, P. E., Tansey, J. T. \& Welte, M. A. PAT proteins, an ancient family of lipid droplet proteins that regulate cellular lipid stores. Biochim. Biophys. Acta 1791, 419-440 2009.

27. Greenberg, A. S. et al. Perilipin, a major hormonally regulated adipocytespecific phosphoprotein associated with the periphery of lipid storage droplets. J. Biol. Chem. 266, 11341-11346 (1991).

28. Hickenbottom, S. J., Kimmel, A. R., Londos, C. \& Hurley, J. H. Structure of a lipid droplet protein; the PAT family member TIP47. Structure 12, 1199-1207 (2004).

29. Kimmel, A. R., Brasaemle, D. L., McAndrews-Hill, M., Sztalryd, C. \& Londos, C. Adoption of PERILIPIN as a unifying nomenclature for the mammalian PAT-family of intracellular lipid storage droplet proteins. J. Lipid Res. 51, 468-471 (2010).

30. Brasaemle, D. L. Thematic review series: adipocyte biology. The perilipin family of structural lipid droplet proteins: stabilization of lipid droplets and control of lipolysis. J. Lipid Res. 48, 2547-2559 (2007).

31. Martinez-Botas, J. et al. Absence of perilipin results in leanness and reverses obesity in Lepr(db/db) mice. Nat. Genet. 26, 474-479 (2000).

32. Tansey, J. T. et al. Perilipin ablation results in a lean mouse with aberrant adipocyte lipolysis, enhanced leptin production, and resistance to diet-induced obesity. Proc. Natl Acad. Sci. USA 98, 6494-6499 (2001).

33. Miyoshi, H. et al. Control of adipose triglyceride lipase action by serine 517 of perilipin A globally regulates protein kinase A-stimulated lipolysis in adipocytes. J. Biol. Chem. 282, 996-1002 (2007).

34. Saha, P. K., Kojima, H., Martinez-Botas, J., Sunehag, A. L. \& Chan, L. Metabolic adaptations in the absence of perilipin: increased beta-oxidation and decreased hepatic glucose production associated with peripheral insulin resistance but normal glucose tolerance in perilipin-null mice. J. Biol. Chem. 279, 35150-35158 (2004)

35. Castro-Chavez, F. et al. Coordinated upregulation of oxidative pathways and downregulation of lipid biosynthesis underlie obesity resistance in perilipin knockout mice: a microarray gene expression profile. Diabetes 52, 2666-2674 (2003).

36. Gong, J., Sun, Z. \& Li, P. CIDE proteins and metabolic disorders. Curr. Opin. Lipidol 20, 121-126 (2009).

37. Xu, L., Zhou, L. \& Li, P. CIDE proteins and lipid metabolism. Arterioscler. Thromb. Vasc. Biol. 32, 1094-1098 (2012).

38. Nishino, N. et al. FSP27 contributes to efficient energy storage in murine white adipocytes by promoting the formation of unilocular lipid droplets. J. Clin. Invest. 118, 2808-2821 (2008).

39. Toh, S. Y. et al. Up-regulation of mitochondrial activity and acquirement of brown adipose tissue-like property in the white adipose tissue of fsp27 deficient mice. PLoS One 3, e2890 (2008).

40. Keller, P. et al. Fat-specific protein 27 regulates storage of triacylglycerol. J. Biol. Chem. 283, 14355-14365 (2008).

41. Puri, V. et al. Fat-specific protein 27, a novel lipid droplet protein that enhances triglyceride storage. J. Biol. Chem. 282, 34213-34218 (2007).

42. Puri, V. et al. Cidea is associated with lipid droplets and insulin sensitivity in humans. Proc. Natl Acad. Sci. USA 105, 7833-7838 (2008).

43. Liu, K. et al. Functional analysis of FSP27 protein regions for lipid droplet localization, caspase-dependent apoptosis, and dimerization with CIDEA. Am. J. Physiol. Endocrinol. Metab. 297, E1395-E1413 (2009).

44. Jambunathan, S., Yin, J., Khan, W., Tamori, Y. \& Puri, V. FSP27 promotes lipid droplet clustering and then fusion to regulate triglyceride accumulation. PLoS One 6, e28614 (2011).
45. Rubio-Cabezas, O. et al. Partial lipodystrophy and insulin resistant diabetes in a patient with a homozygous nonsense mutation in CIDEC. EMBO Mol. Med. 1, 280-287 (2009).

46. Gong, J. et al. Fsp27 promotes lipid droplet growth by lipid exchange and transfer at lipid droplet contact sites. J. Cell Biol. 195, 953-963 (2011).

47. Paar, M. et al. Remodeling Of lipid droplets during lipolysis and growth in adipocytes. J. Biol. Chem. 287, 11164-11173 (2012).

48. Ariotti, N. et al. Postlipolytic insulin-dependent remodeling of micro lipid droplets in adipocytes. Mol. Biol. Cell 23, 1826-1837 (2012).

49. Garcia, A., Sekowski, A., Subramanian, V. \& Brasaemle, D. L. The central domain is required to target and anchor perilipin A to lipid droplets. J. Biol. Chem. 278, 625-635 (2003).

50. Chen, Z., Guo, K., Toh, S. Y., Zhou, Z. \& Li, P. Mitochondria localization and dimerization are required for CIDE-B to induce apoptosis. J. Biol. Chem. 275, 22619-22622 (2000).

51. Wang, H. \& Sztalryd, C. Oxidative tissue: perilipin 5 links storage with the furnace. Trends Endocrinol. Metab. 22, 197-203 (2011).

52. Miyoshi, H. et al. Perilipin overexpression in mice protects against diet-induced obesity. J. Lipid Res. 51, 975-982 (2010).

53. Sawada, T. et al. Perilipin overexpression in white adipose tissue induces a brown fat-like phenotype. PLoS One 5, e14006 (2010).

54. Nian, Z. et al. Fat-specific protein 27 undergoes ubiquitin-dependent degradation regulated by triacylglycerol synthesis and lipid droplet formation. J. Biol. Chem. 285, 9604-9615 (2010).

55. Folch, J., Lees, M. \& Sloane Stanley, G. H. A simple method for the isolation and purification of total lipides from animal tissues. J. Biol. Chem. 226, 497-509 (1957).

56. Ye, J. et al. Cideb, an ER- and lipid droplet-associated protein, mediates VLDL lipidation and maturation by interacting with apolipoprotein B. Cell Metab. 9, 177-190 (2009).

\section{Acknowledgements}

We thank Drs L. Yu and L. Huang in Tsinghua University for their assistance in image analysis and members of P.L.s laboratory for helpful discussion. This work was supported by grants from the National Basic Research Program (2013CB530602 to P.L.), National Natural Science Foundation of China (30925017 and 31030038 to P.L.; 31030020 and 31170679 to M.Y.) and from the Minister of Science and Technology of China (2010AA023002 and 2012CB911101) and Minister of Education (20090002110053).

\section{Author contributions}

Z.S. and J. Gong performed most of the molecular and cell biology experiments, analysed the data and wrote the first draft of the manuscript. H.W., J. Gao and M.Y. performed the crystal structural analysis. M.Y. also helped with some of the experimental design and made significant contribution to data analysis and manuscript preparation. W.X., L.W. and D.X. performed some of the experiments. J-.W.W. and H. Y. helped with experimental design, data analysis and manuscript revision. P.L. is responsible for the experimental design, data coordination, analysis and interpretation, writing, revising and finalization of the manuscript. P.L. is also responsible for the decision to submit the manuscript for publication. All authors read and approved the final manuscript.

\section{Additional information}

Accession codes: Structural coordinates for the N-terminal domain of Fsp27 have been deposited in the Protein Data Bank under accession code 4IKG.

Supplementary Information accompanies this paper at http://www.nature.com/ naturecommunications

Competing financial interests: The authors declare no competing financial interests.

Reprints and permission information is available online at http://npg.nature.com/ reprintsandpermissions/

How to cite this article: Sun, Z. et al. Perilipin1 promotes unilocular lipid droplet formation through the activation of Fsp27 in adipocytes. Nat. Commun. 4:1594 doi: $10.1038 /$ ncomms2581 (2013).

This work is licensed under a Creative Commons AttributionNonCommercial-NoDerivs 3.0 Unported License. To view a copy of this license, visit http://creativecommons.org/licenses/by-nc-nd/3.0/ 


\section{Corrigendum: Perilipin1 promotes unilocular lipid droplet formation through the activation of Fsp27 in adipocytes}

Zhiqi Sun, Jingyi Gong, Han Wu, Wenyi Xu, Lizhen Wu, Dijin Xu, Jinlan Gao, Jia-wei Wu, Hongyuan Yang, Maojun Yang \& Peng Li

Nature Communications 4:1594 doi: 10.1038/ncomms2581 (2013); Published 12 Mar 2013; Updated 17 Sep 2014

The original version of this Article contained an error in the accession codes section, which specified an accession code for an unrelated protein. The correct PDB accession code for structural coordinates of the N-terminal domain of Fsp27 is 4IKG. This has now been corrected in both the PDF and HTML versions of the Article. 